\title{
BLOCKS OF FULLY GRADED RINGS
}

\author{
Everett C. DADE
}

To the memory of Olga Taussky-Todd

\begin{abstract}
We develop Clifford Theory, through the Fong-Reynolds Theorem, for blocks of a ring $\mathfrak{R}$ fully graded by a finite group $G$, with respect to an arbitrary $G$-invariant subring $\mathfrak{S}$ of $\mathfrak{R}$. Our only assumption is that $\mathfrak{R}$ be a finite sum of indecomposable subrings.
\end{abstract}

The writing of this paper was precipitated by a curious observation relating the blocks of a $G$-ring $\mathfrak{T}$, for some finite group $G$, to the blocks of the fixed subring $\mathfrak{T}^{G}$ of $G$ acting on $\mathfrak{T}$. We shall say the an arbitrary ring "has finite block theory" if it is a finite direct sum of indecomposable subrings. The curious observation is that a $G$-ring $\mathfrak{T}$ has finite block theory whenever its fixed subring $\mathfrak{T}^{G}$ has finite block theory. This is easily proved in Theorem 2.2 below. Incidently the converse statement can be false, as Example 2.4 below shows.

A fully $G$-graded ring $\mathfrak{R}$, as defined in $[\mathbf{U}]$ or $\S 4$ below, is a $G$-graded ring whose $\sigma$-components form a group under multiplication. The centralizer of the identity component $\mathfrak{R}_{1}$ in any such $\mathfrak{R}$ is naturally a $G$-ring $\mathfrak{C}$. The fixed subring $\mathfrak{C}^{G}$ of $G$ in $\mathfrak{C}$ is exactly the center $\mathrm{Z}(\mathfrak{R})$ of $\mathfrak{R}$ (see $[\mathbf{U}]$ or $\S 5$ below). This allows us to apply the above observation to $\mathfrak{C}$, concluding in Theorem 5.6 that $\mathfrak{R}$ has finite block theory if and only if $\mathfrak{C}$ does. We can even obtain a one to one correspondence between blocks of $\mathfrak{R}$ and $G$ conjugacy classes of blocks of $\mathfrak{C}$, whether or not $\mathfrak{R}$ has finite block theory (see Theorem 5.8 below).

When the fully $G$-graded ring $\mathfrak{R}$ has finite block theory, we can reproduce Clifford theory for its blocks, as far as the Fong-Reynolds Theorem (Theorem 8.12 below). Following the observation of Ellers $[\mathbf{E}]$ that ordinary Clifford theory works just as well over arbitrary $G$-invariant subalgebras of group algebras, we develop this theory over an arbitary " $G$-invariant" subring $\mathfrak{S}$ of $\mathfrak{R}$ (see $\S 8$ below). One intermediate result in an otherwise straightforward development is surprisingly delicate to prove. It is Theorem 6.6 below, which says that an idempotent $e$ of $\mathfrak{C}$ which is orthogonal to all its $G$-conjugates $e^{\sigma} \neq e$ must lie in the restriction $\mathfrak{R}[H]$ of $\mathfrak{R}$ to its 
stabilizer $H$ in $G$. Once this is out of the way, the rest of Clifford theory is easy.

Perhaps the most interesting part of this paper is to be found in $\S 10$ below, where we speculate about some possible extensions of the theories discussed here. The question of whether our curious observation about $G$-rings has a counterpart for $G$-graded rings is particularly intriguing. We wish we could answer it.

This paper, like the entire journal issue to which it belongs, is dedicated to the memory of the late Olga Taussky-Todd. My first hesitant steps in mathematical research, along with those of so many other young mathematicians, were guided and encouraged by her. I was privileged to coauthor a few papers [DT1], [DT2], [DRTW] with her and others, notably a joint effort [DTZ] with the late Hans Zassenhaus. But she also read over and improved many of my other works. More than forty years ago she introduced me to Max Deuring's Algebren, thus sparking a life-long interest in rings and algebras. The present paper is only the latest consequence of that long ago act.

\section{Finite block theory.}

When we speak of a ring $\mathfrak{R}$, we mean an associative ring with identity element $1=1_{\mathfrak{R}}$. Any subring $\mathfrak{S}$ of $\mathfrak{R}$ must have an identity element $1_{\mathfrak{S}}$, but this identity element need not coincide with $1_{\Re}$. When $1_{\mathfrak{S}}$ is equal to $1_{\mathfrak{R}}$ we say that $\mathfrak{S}$ is a unitary subring of $\mathfrak{R}$. Similary, a homomorphism $\gamma: \mathfrak{R} \rightarrow \mathfrak{T}$ of rings need not send $1_{\mathfrak{R}}$ to $1_{\mathfrak{T}}$. If it does, we say that $\gamma$ is identity-preserving.

Any $\mathfrak{R}$-module $\mathfrak{M}$, whether right, left or two-sided, is understood to be unitary, in the sense that multiplication by $1_{\mathfrak{R}}$ is the identity map of $\mathfrak{M}$ onto itself. We write $\operatorname{Mod}(\mathfrak{R})$ for the abelian category of all right $\mathfrak{R}$-modules and their $\mathfrak{R}$-homomorphisms. If $\mathfrak{M}, \mathfrak{N} \in \operatorname{Mod}(\mathfrak{R})$ (i.e., if $\mathfrak{M}$ and $\mathfrak{N}$ are right $\mathfrak{R}$-modules $)$, then $\operatorname{Hom}_{\mathfrak{R}}(\mathfrak{M}, \mathfrak{N})$ denotes the additive group of all $\mathfrak{R}$ homomorphisms of $\mathfrak{M}$ into $\mathfrak{N}$.

We denote by $\mathrm{Z}(\mathfrak{R})$ the center of a ring $\mathfrak{R}$, and by $\mathrm{ZI}(\mathfrak{R})$ the set of all idempotents in $\mathrm{Z}(\mathfrak{R})$. The set $\mathrm{ZI}(\mathfrak{R})$ is naturally a Boolean algebra, with Boolean operations

$$
e \wedge f=e f=f e, \quad e \vee f=e+f-e f \quad \text { and } \quad e^{\prime}=1-e
$$

for any $e, f \in \mathrm{ZI}(\mathfrak{R})$. The zero element $0=0_{\mathfrak{R}}$ and identity element $1=1_{\mathfrak{R}}$ of the ring $\mathfrak{R}$ are also the zero and identity elements of the Boolean algebra $\mathrm{ZI}(\Re)$. 
The fact that $\mathrm{ZI}(\mathfrak{R})$ is a Boolean algebra gives us two sets of names for most basic properties of idempotents in the ring $\mathrm{Z}(\mathfrak{R})$. Thus two idempotents $e, f$ in $\mathrm{Z}(\mathfrak{R})$ are orthogonal, in the sense that $e f=f e=0$, if and only if they are disjoint in the Boolean algebra ZI $(\Re)$, in the sense that $e \wedge f=f \wedge e=0$. Furthermore an idempotent $e$ is primitive in $\mathrm{Z}(\Re)$, i.e., is non-zero but not the sum of two non-zero orthogonal idempotents in $\mathrm{Z}(\mathfrak{R})$, if and only if it is an atom in the Boolean algebra $\mathrm{ZI}(\mathfrak{R})$, i.e., is non-zero but not the join of two disjoint non-zero elements in $\mathrm{ZI}(\Re)$. Of course distinct primitive idempotents in $\mathrm{Z}(\mathfrak{R})$ are always orthogonal, just as distinct atoms in $\mathrm{ZI}(\mathfrak{R})$ are always disjoint.

We define a block $B$ of a ring $\mathfrak{R}$ to be an ordered pair $(\mathfrak{R}, e)$ consisting of $\mathfrak{R}$ and a primitive idempotent $e$ of $\mathrm{Z}(\mathfrak{R})$. We denote by $\mathrm{Blk}(\mathfrak{R})$ the (possibly empty) set of all such blocks of $\mathfrak{R}$, and by $1_{B}$ the primitive idempotent of $\mathrm{Z}(\mathfrak{R})$ lying in a given block $B=\left(\Re, 1_{B}\right)$ of $\mathfrak{R}$. Thus the $1_{B}$, for $B \in \operatorname{Blk}(\Re)$, are both the distinct primitive idempotents in the ring $\mathrm{Z}(\mathfrak{R})$ and the distinct atoms in the Boolean algebra $\mathrm{ZI}(\mathfrak{R})$. Note that this definition of blocks ensures that two blocks $B$ and $B^{\prime}$ are equal if and only if they are blocks of the same ring $\mathfrak{R}$ corresponding to the same primitive idempotent $1_{B}=1_{B^{\prime}}$ in $\mathrm{Z}(\mathfrak{R})$.

Definition 1.2. A ring $\mathfrak{R}$ has finite block theory if the set $\operatorname{Blk}(\mathfrak{R})$ is finite and $1_{\mathfrak{R}}$ is the finite sum

$$
1_{\mathfrak{R}}=\sum_{B \in \operatorname{Blk}(\mathfrak{R})} 1_{B}
$$

of pairwise orthogonal idempotents in $\mathrm{Z}(\mathfrak{R})$.

There a couple of different ways of expressing this property of $\mathfrak{R}$.

Proposition 1.4. A ring $\mathfrak{R}$ has finite block theory if and only if the set $\mathrm{ZI}(\mathfrak{R})$ of all idempotents in $\mathrm{Z}(\mathfrak{R})$ is finite. This happens if and only if $\mathrm{Blk}(\mathfrak{R})$ is finite and $\mathfrak{R}$ is the finite direct sum

$$
\mathfrak{R}=\sum_{B \in \operatorname{Blk}(\Re)} \mathfrak{R} 1_{B}
$$

of indecomposable subrings.

Proof. If $\mathfrak{R}$ has finite block theory, then $\mathrm{ZI}(\mathfrak{R})$ has a finite number of atoms $1_{B}$, for $B \in \operatorname{Blk}(\mathfrak{R})$. Since these atoms are pairwise disjoint elements in the Boolean algebra $\mathrm{ZI}(\mathfrak{R})$, their sum in $\mathfrak{R}$ is also their join

$$
\bigvee_{B \in \operatorname{Blk}(\mathfrak{R})} 1_{B}=\sum_{B \in \operatorname{Blk}(\mathfrak{R})} 1_{B}=1_{\mathfrak{R}}
$$


in $\mathrm{ZI}(\mathfrak{R})$. So the Boolean algebra $\mathrm{ZI}(\mathfrak{R})$ has a finite number $n \geq 0$ of distinct atoms, and its identity element is the join of those atoms. This implies that $\mathrm{ZI}(\mathfrak{R})$ is isomorphic to the Boolean algebra of all subsets of its set of atoms, and hence has finite order $2^{n}$ (see [B, Chapt. III, Th. 4]).

Conversely, if the Boolean algebra $\mathrm{ZI}(\mathfrak{R})$ is finite, then it has a finite number $n \geq 0$ of distinct atoms $1_{B}$, for $B \in \operatorname{Blk}(\Re)$, and is isomorphic to the Boolean algebra of all subsets of its set of atoms. Hence its identity element is the finite join of its atoms. As above, this is equivalent to (1.3). Therefore $\mathfrak{R}$ has finite block theory if and only if $\mathrm{ZI}(\mathfrak{R})$ is finite.

Since the idempotents $1_{B}$, for $B \in \operatorname{Blk}(\Re)$, are pairwise orthogonal and central in $\mathfrak{R}$, the decomposition (1.3) of $1_{\mathfrak{R}}$ is equivalent to the decomposition $(1.5)$ of $\mathfrak{R}$. The primitivity in $\mathrm{Z}(\mathfrak{R})$ of each idempotent $1_{B}$ in the former decomposition is equivalent to the indecomposability of each subring $\Re 1_{B}$ in the latter decomposition. So the remaining statement of the proposition holds.

Corollary 1.6. If a ring $\Re$ has finite block theory, then the distinct idempotents in $\mathrm{Z}(\mathfrak{R})$ are the finite sums

$$
1_{\mathcal{B}}=\sum_{B \in \mathcal{B}} 1_{B}
$$

where $\mathcal{B}$ runs over all subsets of the finite set $\operatorname{Blk}(\Re)$.

Proof. We follow the convention that empty sums or joins are always zero. So the idempotent $1_{\mathcal{B}}$ in $(1.7)$ is zero when the subset $\mathcal{B}$ of $\operatorname{Blk}(\mathfrak{R})$ is empty.

We saw in the above proof that the Boolean algebra $\mathrm{ZI}(\mathfrak{R})$ is generated by its finite number of atoms $1_{B}$ for $B \in \operatorname{Blk}(\Re)$. It follows that the distinct elements in $\mathrm{ZI}(\mathfrak{R})$ are the joins

$$
1_{\mathcal{B}}=\bigvee_{B \in \mathcal{B}} 1_{B}
$$

where $\mathcal{B}$ runs over all subsets of the finite set $\operatorname{Blk}(\mathfrak{R})$. But this join $1_{\mathcal{B}}$ is equal to the sum on the right side of (1.7) by (1.1), because the atoms $1_{B}$, for $B \in \mathcal{B}$, are pairwise disjoint. Hence the corollary holds.

One final remark about rings with finite block theory is

Proposition 1.8. A ring $\mathfrak{R}$ has finite block theory if and only if its center $\mathrm{Z}(\mathfrak{R})$ has finite block theory.

Proof. The set $\mathrm{ZI}(\mathfrak{R})$ of all idempotents in $\mathrm{Z}(\mathfrak{R})$ is also the set $\mathrm{ZI}(\mathrm{Z}(\mathfrak{R}))$ of all idempotents in $\mathrm{Z}(\mathrm{Z}(\mathfrak{R}))=\mathrm{Z}(\mathfrak{R})$. So the former set is finite if and only if the latter is. By Proposition 1.4 this implies the present proposition. 


\section{G-rings.}

Let $G$ be any multiplicative group. We denote by $1=1_{G}$ the identity element of $G$. We use exponential notation for the conjugation action of $G$ on itself. So conjugation by any $\tau \in G$ sends any element $\sigma \in G$ to $\sigma^{\tau}=\tau^{-1} \sigma \tau \in G$, and any subset $H \subseteq G$ to $H^{\tau}=\tau^{-1} H \tau \subseteq G$.

A $G$-ring $\mathfrak{T}$ is a ring, also called $\mathfrak{T}$, together with an action of the group $G$ as automorphisms of the ring $\mathfrak{T}$. We write the action of $G$ on $\mathfrak{T}$ exponentially, so that any $\sigma \in G$ sends any $t \in \mathfrak{T}$ to $t^{\sigma} \in \mathfrak{T}$. We also use exponential notation for the fixed subring

$$
\mathfrak{T}^{H}=\left\{t \in \mathfrak{T} \mid t^{\sigma}=t, \text { for all } \sigma \in H\right\}
$$

in $\mathfrak{T}$ of any subgroup $H$ of $G$.

A $G$-subring $\mathfrak{S}$ of a $G$-ring $\mathfrak{T}$ is any subring $\mathfrak{S}$ of $\mathfrak{T}$ such that $\mathfrak{S}$ is invariant under the action of $G$ on $\mathfrak{T}$. That action then restricts to one of $G$ on $\mathfrak{S}$, making $\mathfrak{S}$ a $G$-ring in its own right. Evidently the center $\mathrm{Z}(\mathfrak{T})$ is always a unitary $G$-subring of $\mathfrak{T}$.

The following curious result will be our major tool for proving that certain rings have finite block theory.

Theorem 2.2. $\quad$ Let $G$ be a finite multiplicative group, and $\mathfrak{T}$ be a $G$-ring whose fixed subring $\mathfrak{T}^{G}$ has finite block theory. Then $\mathfrak{T}$ has finite block theory.

Proof. The fixed subring $\mathrm{Z}(\mathfrak{T})^{G}$ of the $G$-subring $\mathrm{Z}(\mathfrak{T})$ is clearly a unitary subring of $\mathrm{Z}\left(\mathfrak{T}^{G}\right)$. Since $\mathfrak{T}^{G}$ has finite block theory, its center $\mathrm{Z}\left(\mathfrak{T}^{G}\right)$ has only a finite number of distinct idempotents by Proposition 1.4. Hence there are only a finite number $n$ of distinct idempotents in the subring $\mathrm{Z}(\mathfrak{T})^{G}$ of $\mathrm{Z}\left(\mathfrak{T}^{G}\right)$.

As usual, we write $|S|$ for the cardinality of any set $S$. So $|G|$ is the order of the finite group $G$. Suppose that $\mathfrak{T}$ does not have finite block theory. Then Proposition 1.4 tells us that the Boolean algebra $\mathrm{ZI}(\mathfrak{T})$ of all idempotents in $\mathrm{Z}(\mathfrak{T})$ is infinite. In particular, there is some finite subset $\mathcal{E}$ of $\mathrm{ZI}(\mathfrak{T})$ such that

$$
2^{n|G|}<|\mathcal{E}|
$$

The definition (1.1) of the operations in $\mathrm{ZI}(\mathfrak{T})$ implies that the action of group $G$ on the ring $\mathfrak{T}$ restricts to one of $G$ as automorphisms of the Boolean algebra $\mathrm{ZI}(\mathfrak{T})$. The union

$$
\mathcal{E}^{\prime}=\bigcup_{\sigma \in G} \mathcal{E}^{\sigma}
$$

of the translates of the finite set $\mathcal{E}$ by elements of the finite group $G$ is a finite, $G$-invariant subset of $\mathrm{ZI}(\mathfrak{T})$ satisfying

$$
2^{n|G|}<|\mathcal{E}| \leq\left|\mathcal{E}^{\prime}\right|
$$


The Boolean subalgebra $\mathfrak{B}$ of $\mathrm{ZI}(\mathfrak{T})$ generated by $\mathcal{E}^{\prime}$ is both finite and $G$ invariant. It follows (see [B, Chapt. III, Th. 4]) that it is the full Boolean algebra on its atoms, which form a $G$-invariant subset $\mathcal{F}$ of some finite cardinality $m=|\mathcal{F}|$. Hence $\mathfrak{B}$ has order $2^{m}$. The inclusion $\mathcal{E}^{\prime} \subseteq \mathfrak{B}$ and the above inequalities imply that

$$
2^{n|G|}<\left|\mathcal{E}^{\prime}\right| \leq|\mathfrak{B}|=2^{m}
$$

So we have

$$
n|G|<m=|\mathcal{F}| .
$$

Let $\mathcal{F}_{1}, \mathcal{F}_{2}, \ldots, \mathcal{F}_{h}$ be the distinct $G$-orbits in the finite $G$-set $\mathcal{F}$. Each orbit $\mathcal{F}_{i}$ satisfies

$$
0<\left|\mathcal{F}_{i}\right| \leq|G|
$$

Since $\mathcal{F}$ is the disjoint union of the $\mathcal{F}_{i}$, we conclude that

$$
n|G|<|\mathcal{F}|=\sum_{i=1}^{h}\left|\mathcal{F}_{i}\right| \leq \sum_{i=1}^{h}|G|=h|G| .
$$

Therefore

$$
n<h \text {. }
$$

The members of $\mathcal{F}$ are atoms in the Boolean subalgebra $\mathfrak{B}$ of $\mathrm{ZI}(\mathfrak{T})$. So they are pairwise disjoint non-zero elements in $\mathrm{ZI}(\mathfrak{T})$. This implies that the joins

$$
g_{i}=\bigvee_{f \in \mathcal{F}_{i}} f=\sum_{f \in \mathcal{F}_{i}} f
$$

for $i=1,2, \ldots, h$, are non-zero, pairwise disjoint elements of $\mathrm{ZI}(\mathfrak{R})$. Furthermore, each $g_{i}$ is fixed by $G$, and hence lies in $\mathrm{Z}(\mathfrak{T})^{G}$. So $g_{1}, g_{2}, \ldots g_{h}$ are $h$ distinct idempotents in $\mathrm{Z}(\mathfrak{T})^{G}$. This is impossible, because $h>n$ and $\mathrm{Z}(\mathfrak{T})^{G}$ has exactly $n$ distinct idempotents (see the first paragraph of this proof). Thus the theorem must hold.

The following example shows that the equivalent of the above theorem need not be valid for infinite $G$.

Example 2.3. Let $\mathbb{Z}$ be the ring of all ordinary integers. We form a ring $\mathfrak{T}$ whose additive group is a free $\mathbb{Z}$-module with a basis consisting of the identity element $1=1_{\mathfrak{T}}$ and an infinite number of other elements $e_{i}$, one for each $i \in \mathbb{Z}$. Multiplication in $\mathfrak{T}$ is determined by the rule that

$$
e_{i} e_{j}= \begin{cases}e_{i} & \text { if } i=j, \\ 0 & \text { if } i \neq j\end{cases}
$$


for any $i, j \in \mathbb{Z}$, and the fact that 1 is the two-sided identity element of $\mathfrak{T}$. It is straightforward to verify that $\mathfrak{T}$ is a commutative ring with an infinite number of distinct blocks $\left(\mathfrak{T}, e_{i}\right)$ for $i \in \mathbb{Z}$. So $\mathfrak{T}$ does not have finite block theory.

Let $G$ be the infinite cyclic group $\langle\sigma\rangle$ on one generator $\sigma$. We make $G$ act as automorphisms of the ring $\mathfrak{T}$ so that

$$
\left(e_{i}\right)^{\sigma^{j}}=e_{i+j} \quad \text { and } \quad 1^{\sigma^{j}}=1
$$

for all $i, j \in \mathbb{Z}$. Then $\mathfrak{T}$ becomes a $G$-ring whose fixed subring $\mathfrak{T}^{G}$ just consists of all multiples $n 1_{\mathfrak{T}}$ of $1_{\mathfrak{T}}$ for $n \in \mathbb{Z}$. So $\mathfrak{T}^{G} \simeq \mathbb{Z}$ has finite block theory with just one block $\left(\mathfrak{T}^{G}, 1\right)$, even though $\mathfrak{T}$ does not have finite block theory.

Our next example shows that the converse to Theorem 2.2 need not hold, even when the group $G$ is finite.

Example 2.4. Let $\mathfrak{T}$ and the $e_{i}$, for $i \in \mathbb{Z}$, be as in Example 2.3. We form a two-sided unitary $\mathfrak{T}$-module $\mathfrak{M}$. The additive group of $\mathfrak{M}$ is a free $\mathbb{Z}$ module with a basis consisting of one element $m_{i, j}$ for each pair of elements $i, j \in \mathbb{Z}$ such that $i<j$. The module multiplication in $\mathfrak{M}$ is determined by $\mathbb{Z}$-bilinearity, the fact that right or left multiplication by $1_{\mathfrak{T}}$ must be the identity map of $\mathfrak{M}$ onto itself, and the rule that

$$
e_{i} m_{i^{\prime}, j^{\prime}} e_{j}= \begin{cases}m_{i^{\prime}, j^{\prime}} & \text { if } i=i^{\prime} \text { and } j=j^{\prime} \\ 0 & \text { otherwise }\end{cases}
$$

for any $i, i^{\prime}, j^{\prime}, j \in \mathbb{Z}$ with $i^{\prime}<j^{\prime}$.

We make the direct sum $\mathfrak{T} \oplus \mathfrak{M}$ of the additive groups $\mathfrak{T}$ and $\mathfrak{M}$ into a ring with the multiplication given by

$$
(t \oplus m)\left(t^{\prime} \oplus m^{\prime}\right)=\left(t t^{\prime}\right) \oplus\left(t m^{\prime}+m t^{\prime}\right)
$$

for any $t, t^{\prime} \in \mathfrak{T}$ and $m, m^{\prime} \in \mathfrak{M}$. Here, of course, $t t^{\prime}$ is computed in the ring $\mathfrak{T}$, and $t m^{\prime}+m t^{\prime}$ in the two-sided $\mathfrak{T}$-module $\mathfrak{M}$. It is straightforward to verify that

$$
\mathrm{Z}(\mathfrak{T} \oplus \mathfrak{M})=\mathbb{Z}\left(1_{\mathfrak{T}} \oplus 0_{\mathfrak{M}}\right)=\mathbb{Z} 1_{\mathfrak{T} \oplus \mathfrak{M}} \simeq \mathbb{Z} .
$$

So $\mathfrak{T} \oplus \mathfrak{M}$ has finite block theory, with $(\mathfrak{T} \oplus \mathfrak{M}, 1)$ as its only block.

Let $G$ be the cyclic group $\langle\tau\rangle$ generated by an element $\tau$ of order two. We make $G$ act as automorphisms of the ring $\mathfrak{T} \oplus \mathfrak{M}$ so that

$$
(t \oplus m)^{\tau}=t \oplus(-m)
$$


for any $t \in \mathfrak{T}$ and $m \in \mathfrak{M}$. Then the fixed subring $(\mathfrak{T} \oplus \mathfrak{M})^{G}$ in the $G$-ring $\mathfrak{T} \oplus \mathfrak{M}$ is $\mathfrak{T} \oplus 0$, which is isomorphic to $\mathfrak{T}$ as a ring. We know from the discussion in Example 2.3 that the ring $\mathfrak{T}$ has an infinite number of blocks. Hence the isomorphic ring $(\mathfrak{T} \oplus \mathfrak{M})^{G}$ does not have finite block theory, even though $\mathfrak{T} \oplus \mathfrak{M}$ has finite block theory.

\section{G-graded rings.}

Let $G$ be any multiplicative group. A $G$-graded ring $\Re$ is a ring, also denoted by $\mathfrak{R}$, together with a decomposition

$$
\mathfrak{R}=\sum_{\sigma \in G} \mathfrak{R}_{\sigma}
$$

of $\mathfrak{R}$ as a direct sum of additive subgroups $\mathfrak{R}_{\sigma}$, whose products in the ring $\mathfrak{R}$ satisfy

$$
\mathfrak{R}_{\sigma} \mathfrak{R}_{\tau} \subseteq \mathfrak{R}_{\sigma \tau}
$$

for all $\sigma, \tau \in G$. The decomposition (3.1a) is the $G$-grading of $\Re$, and the additive subgroup $\mathfrak{R}_{\sigma}$ is the $\sigma$-component of $\mathfrak{R}$ for any $\sigma \in G$. We always have

$$
1_{\mathfrak{R}} \in \mathfrak{R}_{1} .
$$

So the identity component $\mathfrak{R}_{1}$ is a unitary subring of $\mathfrak{R}$, and each $\sigma$-component $\mathfrak{R}_{\sigma}$ is a two-sided $\mathfrak{R}_{1}$-submodule of $\mathfrak{R}$.

For the rest of this section we fix a multiplicative group $G$ and a $G$-graded ring $\mathfrak{R}$. Given a subset $H \subseteq G$, we denote by $\mathfrak{R}[H]$ the additive subgroup

$$
\mathfrak{R}[H]=\sum_{\sigma \in H} \mathfrak{R}_{\sigma}
$$

of $\mathfrak{R}$. By convention $\mathfrak{R}[H]$ is zero when $H$ is empty. If $H$ is a subgroup of $G$, then $\mathfrak{R}[H]$ is a unitary subring of $\mathfrak{R}$. In that case the $H$-grading (3.3a) turns $\mathfrak{R}[H]$ into an $H$-graded ring with the $\sigma$-component

$$
\mathfrak{R}[H]_{\sigma}=\mathfrak{R}_{\sigma}
$$

for any $\sigma \in H$. We call this $H$-graded ring $\mathfrak{R}[H]$ the restriction of $\mathfrak{R}$ to $H$.

Let $H$ be a subgroup of $G$. An $H$-graded subring $\mathfrak{S}$ of $\mathfrak{R}$ is any subring $\mathfrak{S}$ of the ring $\mathfrak{R}$ such that

$$
\mathfrak{S}=\sum_{\sigma \in H}\left(\mathfrak{S} \cap \mathfrak{R}_{\sigma}\right)
$$


Such an $\mathfrak{S}$ is itself an $H$-graded ring with the $\sigma$-component

$$
\mathfrak{S}_{\sigma}=\mathfrak{S} \cap \mathfrak{R}_{\sigma}
$$

for any $\sigma \in H$. Clearly $\mathfrak{R}[H]$ is the unique largest $H$-graded subring of $\mathfrak{R}$.

For our purposes the most important subring of $\mathfrak{R}$ is the centralizer

$$
\mathfrak{C}=\mathrm{C}_{\mathfrak{R}}\left(\mathfrak{R}_{1}\right)=\left\{c \in \mathfrak{R} \mid c r_{1}=r_{1} c \text { for all } r_{1} \in \mathfrak{R}_{1}\right\}
$$

of the identity component $\mathfrak{R}_{1}$. It follows easily from (3.1) that:

The centralizer $\mathfrak{C}$ is a unitary $G$-graded subring of $\mathfrak{R}$.

For any $\sigma \in G$ the $\sigma$-component of $\mathfrak{C}$ is the centralizer

$$
\begin{aligned}
& \mathfrak{C}_{\sigma}=\mathrm{C}\left(\mathfrak{R}_{1} \text { in } \mathfrak{R}_{\sigma}\right)=\left\{c \in \mathfrak{R}_{\sigma} \mid c r_{1}=r_{1} c \quad \text { for all } r_{1} \in \mathfrak{R}_{1}\right\} \\
& \quad \text { of } \mathfrak{R}_{1} \text { in } \mathfrak{R}_{\sigma} .
\end{aligned}
$$

In particular, the identity component of $\mathfrak{C}$ is the center

$$
\mathfrak{C}_{1}=\mathrm{C}\left(\mathfrak{R}_{1} \text { in } \mathfrak{R}_{1}\right)=\mathrm{Z}\left(\Re_{1}\right)
$$

of $\mathfrak{R}_{1}$. Since $\mathfrak{C}_{1} \subseteq \mathfrak{R}_{1}$ centralizes $\mathfrak{C}=\mathrm{C}_{\mathfrak{R}}\left(\mathfrak{R}_{1}\right)$, and contains the common identity element $1_{\mathfrak{R}}$ of $\mathfrak{C}$ and $\mathfrak{R}_{1}$, we have:

$$
\begin{aligned}
& \text { The identity component } \mathfrak{C}_{1} \text { is a unitary central subring } \\
& \text { of both } \mathfrak{R}_{1} \text { and } \mathfrak{C} .
\end{aligned}
$$

If $H$ is any subgroup of $G$, then $H \backslash G$ (which we pronounce as " $H$ under $G$ ") will denote the set of all left cosets $H \tau$ of $H$ in $G$. The group $G$ acts on the set $H \backslash G$, with any $\sigma \in G$ sending any $T \in H \backslash G$ to $T \sigma \in H \backslash G$. An $H \backslash G$-graded $\mathfrak{R}$-module $\mathfrak{M}$ is a right $\mathfrak{R}$-module, also called $\mathfrak{M}$, together with a decomposition

$$
\mathfrak{M}=\sum_{T \in H \backslash G} \mathfrak{M}_{T}
$$

of $\mathfrak{M}$ as a direct sum of additive subgroups $\mathfrak{M}_{T}$, whose products with the $\sigma$-components of $\mathfrak{R}$ satisfy

$$
\mathfrak{M}_{T} \mathfrak{R}_{\sigma} \subseteq \mathfrak{M}_{T \sigma}
$$

for any $T \in H \backslash G$ and $\sigma \in G$. The decomposition (3.9a) is the $H \backslash G$-grading of $\mathfrak{M}$, and the additive subgroup $\mathfrak{M}_{T}$ is the $T$-component of $\mathfrak{M}$ for any $T \in H \backslash G$. 
An $H \backslash G$-graded $\mathfrak{R}$-submodule $\mathfrak{N}$ of an $H \backslash G$-graded $\mathfrak{R}$-module $\mathfrak{M}$ is any $\mathfrak{R}$-submodule $\mathfrak{N}$ of the $\mathfrak{R}$-module $\mathfrak{M}$ such that

$$
\mathfrak{N}=\sum_{T \in H \backslash G}\left(\mathfrak{M}_{T} \cap \mathfrak{N}\right)
$$

Any such $\mathfrak{N}$ is itself an $H \backslash G$-graded $\mathfrak{R}$-module, with the $T$-component

$$
\mathfrak{N}_{T}=\mathfrak{M}_{T} \cap \mathfrak{N}
$$

for any $T \in H \backslash G$. Furthermore, the factor $\mathfrak{R}$-module $\mathfrak{M} / \mathfrak{N}$ is naturally $H \backslash G$-graded, with the $T$-component

$$
(\mathfrak{M} / \mathfrak{N})_{T}=\left(\mathfrak{M}_{T}+\mathfrak{N}\right) / \mathfrak{N} \simeq \mathfrak{M}_{T} / \mathfrak{N}_{T}
$$

for any $T \in H \backslash G$.

We form a category $\operatorname{GrMod}(H \backslash G, \mathfrak{R})$, whose objects are the $H \backslash G$-graded $\mathfrak{R}$-modules, and whose morphisms $\phi: \mathfrak{M} \rightarrow \mathfrak{N}$ are those homomorphisms of the underlying $\mathfrak{R}$-modules which preserve $H \backslash G$-gradings, in the sense that

$$
\phi\left(\mathfrak{M}_{T}\right) \subseteq \mathfrak{N}_{T}
$$

for any $T \in H \backslash G$. For fixed objects $\mathfrak{M}, \mathfrak{N} \in \operatorname{GrMod}(H \backslash G, \mathfrak{R})$, the morphisms $\phi: \mathfrak{M} \rightarrow \mathfrak{N}$ in $\operatorname{GrMod}(H \backslash G, \mathfrak{R})$ form a group $\operatorname{GrHom}_{H \backslash G, \mathfrak{R}}(\mathfrak{M}, \mathfrak{N})$ under addition of module homomorphisms. It follows that $\operatorname{GrMod}(H \backslash G, \mathfrak{R})$ is an additive category, with composition of maps as multiplication.

We indicate that $\phi: \mathfrak{M} \rightarrow \mathfrak{N}$ is a morphism in $\operatorname{GrMod}(H \backslash G, \mathfrak{R})$ by saying that it is a homomorphism of $H \backslash G$-graded $\mathfrak{R}$-modules. In that case the kernel

$$
\operatorname{ker}(\phi)=\{m \in \mathfrak{M} \mid \phi(m)=0\}
$$

of $\phi$ is an $H \backslash G$-graded $\mathfrak{R}$-submodule of $\mathfrak{M}$, and the image $\phi(\mathfrak{M})$ of $\phi$ is an $H \backslash G$-graded $\mathfrak{R}$-submodule of $\mathfrak{N}$. It follows that the injection of $\operatorname{ker}(\phi)$ into $\mathfrak{M}$ and the projection of $\mathfrak{N}$ onto $\mathfrak{N} / \phi(\mathfrak{M})$ are a kernel and cokernel, respectively, for the morphism $\phi$ in the category $\operatorname{GrMod}(H \backslash G, \mathfrak{R})$. With this observation it is easy to see that $\operatorname{GrMod}(H \backslash G, \mathfrak{R})$ is an abelian category, in which monomorphisms, epimorphisms and isomorphisms are just those morphisms which are monomorphisms, epimorphisms or isomorphisms, respectively, of the underlying $\mathfrak{R}$-modules.

The inclusions (3.9b) imply that the $H$-component of any $H \backslash G$-graded $\mathfrak{R}$-module $\mathfrak{M}$ is an $\mathfrak{R}[H]$-submodule $\mathfrak{M}_{H}$ of $\mathfrak{M}$. Furthermore, any homomorphism $\phi: \mathfrak{M} \rightarrow \mathfrak{N}$ of $H \backslash G$-graded $\mathfrak{R}$-modules restricts to a homomorphism $\phi_{H}: \mathfrak{M}_{H} \rightarrow \mathfrak{N}_{H}$ of right $\mathfrak{R}[H]$-modules. It follows that restriction to $H$ components is an additive functor $(\cdot)_{H}$ from $\operatorname{GrMod}(H \backslash G, \mathfrak{R})$ to $\operatorname{Mod}(\mathfrak{R}[H])$. 
Since $G$ is the disjoint union of the cosets $T \in H \backslash G$, the $G$-graded ring $\mathfrak{R}$ is the direct sum

$$
\mathfrak{R}=\sum_{T \in H \backslash G} \mathfrak{R}[T]
$$

of additive subgroups. It follows easily from (3.1b) and (3.3a) that

$$
\mathfrak{R}[T] \mathfrak{R}_{\sigma} \subseteq \mathfrak{R}[T \sigma]
$$

for any $T \in H \backslash G$ and $\sigma \in G$. So the $H \backslash G$-grading (3.13a) turns the regular right $\mathfrak{R}$-module $\mathfrak{R}$ into an $H \backslash G$-graded $\mathfrak{R}$-module.

Any right $\mathfrak{R}[H]$-module $\mathfrak{K}$ induces a right $\mathfrak{R}$-module $\mathfrak{K} \otimes \mathfrak{R}=\mathfrak{K} \otimes_{\mathfrak{R}[H]} \mathfrak{R}$. Each coset $T=H \tau \in H \backslash G$ is closed under left multiplication by elements of $H$. Hence the corresponding summand $\mathfrak{R}[T]$ in (3.13a) is a left $\mathfrak{R}[H]$ submodule of $\mathfrak{R}$. It follows that $\mathfrak{K} \otimes \mathfrak{R}$ is, after natural identifications, the direct sum

$$
\mathfrak{K} \otimes \mathfrak{R}=\sum_{T \in H \backslash G} \mathfrak{K} \otimes \mathfrak{R}[T]
$$

of additive subgroups. In view of (3.13b) we have

$$
(\mathfrak{K} \otimes \mathfrak{R}[T]) \mathfrak{R}_{\sigma} \subseteq \mathfrak{K} \otimes \mathfrak{R}[T \sigma]
$$

for any $T \in H \backslash G$ and $\sigma \in G$. Hence the $H \backslash G$-grading (3.14a) turns $\mathfrak{K} \otimes \mathfrak{R}$ into an $H \backslash G$-graded $\mathfrak{R}$-module. Any homomorphism $\psi: \mathfrak{K} \rightarrow \mathfrak{L}$ of right $\mathfrak{R}[H]$-modules induces a homomorphism $\psi \otimes \mathfrak{R}: \mathfrak{K} \otimes \mathfrak{R} \rightarrow \mathfrak{L} \otimes \mathfrak{R}$ of $H \backslash G$ graded $\mathfrak{R}$-modules, sending $k \otimes r$ to $\psi(k) \otimes r$ for any $k \in \mathfrak{K}$ and $r \in \mathfrak{R}$. In this way induction from $\mathfrak{R}[H]$ to $\mathfrak{R}$ becomes an additive functor $\bullet \otimes \mathfrak{R}$ from $\operatorname{Mod}(\mathfrak{R}[H])$ to $\operatorname{GrMod}(H \backslash G, \mathfrak{R})$.

The composite functor $(\cdot \otimes \mathfrak{R})_{H}: \operatorname{Mod}(\mathfrak{R}[H]) \rightarrow \operatorname{Mod}(\mathfrak{R}[H])$ sends any right $\mathfrak{R}[H]$-module $\mathfrak{K}$ to the $H$-component $\mathfrak{K} \otimes \mathfrak{R}[H]$ of the induced $H \backslash G$ graded $\mathfrak{R}$-module $\mathfrak{K} \otimes \mathfrak{R}$. Since these tensor products are over $\mathfrak{R}[H]$, the natural map

$$
\gamma_{\mathfrak{K}}: k \mapsto k \otimes 1_{\mathfrak{R}}
$$

is an isomorphism of $\mathfrak{K}$ onto $\mathfrak{K} \otimes \mathfrak{R}[H]$ as right $\mathfrak{R}[H]$-modules. It is straightforward to verify that:

The above isomorphisms $\gamma_{\mathfrak{K}}$ form a natural equivalence $\gamma$

between the identity functor on the category $\operatorname{Mod}(\mathfrak{R}[H])$ and the composite functor $(\cdot \otimes \mathfrak{R})_{H}: \operatorname{Mod}(\mathfrak{R}[H]) \rightarrow \operatorname{Mod}(\mathfrak{R}[H])$. 


\section{Fully graded rings.}

By a fully $G$-graded ring $\mathfrak{R}$ we mean a $G$-graded ring $\mathfrak{R}$ in which the inclusion (3.1b) is equality

$$
\mathfrak{R}_{\sigma} \mathfrak{R}_{\tau}=\mathfrak{R}_{\sigma \tau}
$$

for any $\sigma, \tau \in G$. Such rings are also called strongly G-graded (see [D]). Notice that the product $\mathfrak{R}_{\sigma} \mathfrak{R}_{\tau}$ in (4.1), like all our products of additive subgroups of $\mathfrak{R}$, is the additive subgroup of $\mathfrak{R}$ generated by the products $r_{\sigma} r_{\tau}^{\prime} \in \mathfrak{R}$ of elements $r_{\sigma} \in \mathfrak{R}_{\sigma}$ and $r_{\tau}^{\prime} \in \mathfrak{R}_{\tau}$, and not just the set of those products.

For the rest of this paper we assume that:

Hypothesis 4.2. $G$ is an arbitrary multiplicative group, and $\mathfrak{R}$ is a fully G-graded ring.

Our main interest is in the case of finite $G$. But enough results hold for arbitrary $G$ to make it undesirable to restrict ourselves just to finite groups.

The following consequence of (4.1) has many uses.

Lemma 4.3. Given any $\tau \in G$, there exist a finite number $n \geq 1$ of elements $s_{1}, s_{2}, \ldots, s_{n} \in \mathfrak{R}_{\tau^{-1}}$ and $t_{1}, t_{2}, \ldots, t_{n} \in \mathfrak{R}_{\tau}$ such that

$$
1_{\Re}=s_{1} t_{1}+s_{2} t_{2}+\cdots+s_{n} t_{n}
$$

Proof. Equation (4.1) for $\sigma=\tau^{-1}$ is

$$
\mathfrak{R}_{1}=\mathfrak{R}_{\tau^{-1}} \mathfrak{R}_{\tau}
$$

Since $1_{\mathfrak{R}}$ lies in $\mathfrak{R}_{1}$ by $(3.2)$, this implies the lemma.

We first apply the above lemma in the study of the structure of $\mathfrak{R}$ as a module over its restriction to a subgroup $H$ of $G$. Specifically, we use it to prove:

Lemma 4.4. Suppose that elements $s_{1}, s_{2}, \ldots, s_{n}$ and $t_{1}, t_{2}, \ldots, t_{n}$ satisfy the conditions in Lemma 4.3 for some $\tau \in G$. For any subgroup $H$ of $G$, the left $\mathfrak{R}[H]$-submodule $\mathfrak{R}[H \tau]$ of $\mathfrak{R}$ is projective and generated by $t_{1}, t_{2}, \ldots, t_{n}$. Similarly, the right $\mathfrak{R}[H]$-submodule $\mathfrak{R}\left[\tau^{-1} H\right]$ of $\mathfrak{R}$ is projective and generated by $s_{1}, s_{2}, \ldots, s_{n}$. 
Proof. By left-right symmetry we only need prove the lemma for $\mathfrak{R}[H \tau]$. The fact that the subset $H \tau$ of $G$ is closed under left multiplication by elements of $H$ implies that $\mathfrak{R}[H \tau]$ is a left $\mathfrak{R}[H]$-submodule of $\mathfrak{R}$. Since $s_{i} \in \mathfrak{R}_{\tau^{-1}}$ for each $i=1,2, \ldots, n$, we have

$$
r s_{i} \in \mathfrak{R}[H \tau] \mathfrak{R}_{\tau^{-1}} \subseteq \mathfrak{R}\left[H \tau \tau^{-1}\right]=\mathfrak{R}[H]
$$

for any $r \in \mathfrak{R}[H \tau]$. It follows that the map

$$
\Phi: r \mapsto\left(r s_{1}, r s_{2}, \ldots, r s_{n}\right)
$$

is a homomorphism of the left $\mathfrak{R}[H]$-module $\mathfrak{R}[H \tau]$ into the external direct sum

$$
\mathfrak{R}[H]^{(n)}=\mathfrak{R}[H] \oplus \mathfrak{R}[H] \oplus \cdots \oplus \mathfrak{R}[H]
$$

of $n$ copies of the regular left $\mathfrak{R}[H]$-module $\mathfrak{R}[H]$. Similarly, the product $u t_{i}$ lies in $\mathfrak{R}[H] \mathfrak{R}_{\tau} \subseteq \mathfrak{R}[H \tau]$ for any $u \in \mathfrak{R}[H]$ and $i=1,2, \ldots, n$. Hence the map

$$
\Psi:\left(u_{1}, u_{2}, \ldots, u_{n}\right) \mapsto u_{1} t_{1}+u_{2} t_{2}+\cdots+u_{n} t_{n}
$$

is a homomorphism of $\mathfrak{R}[H]^{(n)}$ into $\mathfrak{R}[H \tau]$ as left $\mathfrak{R}[H]$-modules. The composite homomorphism $\Psi \Phi: \mathfrak{R}[H \tau] \rightarrow \mathfrak{R}[H \tau]$ is the identity map, because

$\Psi(\Phi(r))=r s_{1} t_{1}+r s_{2} t_{2}+\cdots+r s_{n} t_{n}=r\left(s_{1} t_{1}+s_{2} t_{2}+\cdots+s_{n} t_{n}\right)=r 1=r$

for any $r \in \mathfrak{R}[H \tau]$. We conclude that $\mathfrak{R}[H \tau]$ is generated by $t_{1}, t_{2}, \ldots, t_{n}$ as a left $\mathfrak{R}[H]$-module, and is isomorphic to a direct summand of the free left $\mathfrak{R}[H]$-module $\mathfrak{R}[H]^{(n)}$. So it is a projective left $\mathfrak{R}[H]$-module, and the lemma is proved.

What we really need is the following consequence of the preceding lemma.

Proposition 4.5. If Hypothesis 4.2 holds, then $\mathfrak{R}$ is projective as both a left and a right module over its restriction $\mathfrak{R}[H]$ to any subgroup $H$ of $G$.

Proof. By left-right symmetry we only need prove the proposition for the left $\mathfrak{R}[H]$-module $\mathfrak{R}$. Because $G$ is the disjoint union of the left cosets $T=$ $H \tau \in H \backslash G$, the left $\mathfrak{R}[H]$-module $\mathfrak{R}$ is the direct sum

$$
\mathfrak{R}=\sum_{T \in H \backslash G} \mathfrak{R}[T]
$$

of left $\mathfrak{R}[H]$-submodules. Lemma 4.4 tells us that each summand $\mathfrak{R}[T]=$ $\mathfrak{R}[H \tau]$ in this decomposition is a projective left $\mathfrak{R}[H]$-module. Hence so is their direct sum $\mathfrak{R}$. Thus the proposition holds. 
There is an equality similar to (4.1) for components of $H \backslash G$-graded $\mathfrak{R}$ modules.

Lemma 4.6. If $\mathfrak{M}$ is an $H \backslash G$-graded $\mathfrak{R}$-module, then

$$
\mathfrak{M}_{T} \mathfrak{R}_{\sigma}=\mathfrak{M}_{T \sigma}
$$

for any $T \in H \backslash G$ and $\sigma \in G$.

Proof. We know from $(3.9 \mathrm{~b})$ that

$$
\mathfrak{M}_{T} \mathfrak{R}_{\sigma} \subseteq \mathfrak{M}_{T \sigma}
$$

Since

$$
1_{\mathfrak{R}} \in \mathfrak{R}_{1}=\mathfrak{R}_{\sigma^{-1}} \mathfrak{R}_{\sigma}
$$

by (3.2) and (4.1), we have

$$
\mathfrak{M}_{T \sigma}=\left(\mathfrak{M}_{T \sigma}\right) 1_{\mathfrak{R}} \subseteq \mathfrak{M}_{T \sigma} \mathfrak{R}_{\sigma^{-1}} \mathfrak{R}_{\sigma} \subseteq \mathfrak{M}_{T \sigma \sigma^{-1}} \mathfrak{R}_{\sigma}=\mathfrak{M}_{T} \mathfrak{R}_{\sigma}
$$

Hence the lemma holds.

We apply the above lemma to prove:

Proposition 4.7. If Hypothesis 4.2 holds and $H$ is any subgroup of $G$, then a homomorphism $\phi: \mathfrak{M} \rightarrow \mathfrak{N}$ of $H \backslash G$-graded $\mathfrak{R}$-modules is a monomorphism, epimorphism or isomorphism if and only if its restriction $\phi_{H}: \mathfrak{M}_{H} \rightarrow \mathfrak{N}_{H}$ to $H$-components is a monomorphism, epimorphism or isomorphism, respectively.

Proof. The kernel of $\phi$ is an $H \backslash G$-graded $\mathfrak{R}$-submodule $\mathfrak{K}$ of $\mathfrak{M}$. Its $H$ component $\mathfrak{K}_{H}$ is the kernel of the restriction $\phi_{H}$ of $\phi$ to $\mathfrak{M}_{H}$. Clearly $\mathfrak{K}_{H}=0$ when $\mathfrak{K}=0$. Conversely, if $\mathfrak{K}_{H}=0$, then Lemma 4.6 for the $H \backslash G$-graded $\mathfrak{R}$-module $\mathfrak{K}$ implies that

$$
\mathfrak{K}_{T}=\mathfrak{K}_{H \sigma}=\mathfrak{K}_{H} \mathfrak{R}_{\sigma}=0
$$

for any $\operatorname{coset} T=H \sigma \in H \backslash G$. Hence

$$
\mathfrak{K}=\sum_{T \in H \backslash G} \mathfrak{K}_{T}=0 .
$$

Thus we have shown that $\mathfrak{K}=0$ if and only if $\mathfrak{K}_{H}=0$, i.e., that $\phi$ is a monomorphism if and only if $\phi_{H}$ is a monomorphism. 
The image of $\phi$ is an $H \backslash G$-graded $\mathfrak{R}$-submodule $\mathfrak{L}$ of $\mathfrak{N}$. Its $H$-component $\mathfrak{L}_{H}$ is the image of $\phi_{H}$. The $H \backslash G$-graded factor $\mathfrak{R}$-module $\mathfrak{N} / \mathfrak{L}$ has an $H$-component $(\mathfrak{N} / \mathfrak{L})_{H}$ isomorphic to $\mathfrak{N}_{H} / \mathfrak{L}_{H}$. So $\mathfrak{N}_{H} / \mathfrak{L}_{H}=0$ whenever $\mathfrak{N} / \mathfrak{L}=0$. Conversely, if $\mathfrak{N}_{H} / \mathfrak{L}_{H}=0$, then $(\mathfrak{N} / \mathfrak{L})_{H}=0$. As above, this and Lemma 4.6 for the $H \backslash G$-graded $\mathfrak{R}$-module $\mathfrak{N} / \mathfrak{L}$ imply that $\mathfrak{N} / \mathfrak{L}=0$. Thus we have shown that $\mathfrak{N} / \mathfrak{L}=0$ if and only if $\mathfrak{N}_{H} / \mathfrak{L}_{H}=0$, i.e., that $\phi$ is an epimorphism if and only if $\phi_{H}$ is an epimorphism.

The homomorphism $\phi$ is an isomorphism if and only if it is both a monomorphism and an epimorphism. We have seen above that this happens if and only if $\phi_{H}$ is both a monomorphism and an epimorphism, i.e., if and only if $\phi_{H}$ is an isomorphism. So the proposition is proved.

Any $H \backslash G$-graded $\mathfrak{R}$-module $\mathfrak{M}$ restricts to a right $\mathfrak{R}[H]$-module $\mathfrak{M}_{H}$, from which we can form the induced $H \backslash G$-graded $\mathfrak{R}$-module $\left(\mathfrak{M}_{H}\right) \otimes \mathfrak{R}$ as in (3.14). There is a natural homomorphism $\delta_{\mathfrak{M}}$ of the $\mathfrak{R}$-module $\left(\mathfrak{M}_{H}\right) \otimes \mathfrak{R}$ into $\mathfrak{M}$, sending $m \otimes r$ to

$$
\delta_{\mathfrak{M}}(m \otimes r)=m r \in \mathfrak{M}
$$

for any $m \in \mathfrak{M}_{H}$ and $r \in \mathfrak{R}$. We use these homomorphisms in the following explicit version of [NRvO, 3.12].

Proposition 4.9. If Hypothesis 4.2 holds and $H$ is any subgroup of $G$, then the map $\delta_{\mathfrak{M}}:\left(\mathfrak{M}_{H}\right) \otimes \mathfrak{R} \rightarrow \mathfrak{M}$ is an isomorphism of $H \backslash G$-graded $\mathfrak{R}$ modules for any $\mathfrak{M} \in \operatorname{GrMod}(H \backslash G, \mathfrak{R})$. These isomorphisms form a natural equivalence $\delta$ between the composite functor

$$
(\cdot)_{H} \otimes \mathfrak{R}: \operatorname{GrMod}(H \backslash G, \mathfrak{R}) \rightarrow \operatorname{GrMod}(H \backslash G, \mathfrak{R})
$$

and the identity functor on $\operatorname{GrMod}(H \backslash G, \mathfrak{R})$. Hence the two functors

$$
(\cdot)_{H}: \operatorname{Gr} \operatorname{Mod}(H \backslash G, \mathfrak{R}) \rightarrow \operatorname{Mod}(\mathfrak{R}[H])
$$

and

$$
\cdot \otimes \mathfrak{R}: \operatorname{Mod}(\mathfrak{R}[H]) \rightarrow \operatorname{GrMod}(H \backslash G, \mathfrak{R})
$$

form an equivalence between the abelian categories $\operatorname{GrMod}(H \backslash G, \mathfrak{R})$ and $\operatorname{Mod}(\Re[H])$.

Proof. The $\mathfrak{R}$-homomorphism $\delta_{\mathfrak{M}}$ sends the $T$-component $\left(\mathfrak{M}_{H}\right) \otimes \mathfrak{R}[T]$ of $\left(\mathfrak{M}_{H}\right) \otimes \mathfrak{R}$ onto the additive subgroup $\mathfrak{M}_{H} \mathfrak{R}[T]$ of $\mathfrak{M}$ for any $T \in H \backslash G$. It follows from $(3.9 \mathrm{~b})$ that

$$
\mathfrak{M}_{H} \mathfrak{R}[T]=\sum_{\sigma \in T} \mathfrak{M}_{H} \mathfrak{R}_{\sigma} \subseteq \sum_{\sigma \in T} \mathfrak{M}_{H \sigma} .
$$


Since $H \sigma=T$ for any $\sigma$ in the left coset $T$ of $H$, this last sum is just $\mathfrak{M}_{T}$. Hence $\delta_{\mathfrak{M}}$ sends $\left(\mathfrak{M}_{H}\right) \otimes \mathfrak{R}[T]$ into $\mathfrak{M}_{T}$. So $\delta_{\mathfrak{M}}:\left(\mathfrak{M}_{H}\right) \otimes \mathfrak{R} \rightarrow \mathfrak{M}$ is a homomorphism of $H \backslash G$-graded $\mathfrak{R}$-modules. The restriction of $\delta_{\mathfrak{M}}$ to $H$ components is the natural isomorphism of $\left(\mathfrak{M}_{H}\right) \otimes \mathfrak{R}[H]=\left(\mathfrak{M}_{H}\right) \otimes_{\mathfrak{R}[H]} \mathfrak{R}[H]$ onto the right $\mathfrak{R}[H]$-module $\mathfrak{M}_{H}$. We conclude from this and Proposition 4.7 that $\delta_{\mathfrak{M}}$ is an isomorphism. Thus the first statement of the proposition holds.

It is straightforward to verify that the $\delta_{\mathfrak{M}}$ form a natural transformation $\delta$ of the composite functor $(\cdot)_{H} \otimes \mathfrak{R}$ into the identity functor on $\operatorname{GrMod}(H \backslash G, \mathfrak{R})$. Because each $\delta_{\mathfrak{M}}$ is an isomorphism, this natural transformation is a natural equivalence. We know from (3.15b) that the other composite functor $(\cdot \otimes \mathfrak{R})_{H}$ is naturally equivalent to the identity functor on $\operatorname{Mod}(\mathfrak{R}[H])$. Therefore the remaining statements of the proposition hold.

\section{Centralizers of identity components.}

As in (3.5) we define $\mathfrak{C}$ to be the unitary $G$-graded subring $\mathrm{C}_{\mathfrak{R}}\left(\mathfrak{R}_{1}\right)$ of our fully $G$-graded ring $\mathfrak{R}$. There is a natural action of the group $G$ as automorphisms of the ring $\mathfrak{C}$ (see $[\mathbf{U}]$ ). Since we're going to need various properties of this action, we discuss its definition in detail.

Lemma 5.1. If $c \in \mathfrak{C}$ and $\tau \in G$, then there is a unique element $c^{\tau} \in \mathfrak{R}$ such that

$$
r_{\tau} c^{\tau}=c r_{\tau}
$$

for all $r_{\tau} \in \mathfrak{R}_{\tau}$. This element $c^{\tau}$ is given by

$$
c^{\tau}=\sum_{i=1}^{n} s_{i} c t_{i} \in \mathfrak{R}
$$

whenever $s_{1}, s_{2}, \ldots, s_{n}$ and $t_{1}, t_{2}, \ldots, t_{n}$ satisfy the conditions in Lemma 4.3.

Proof. Suppose that $c^{\tau} \in \mathfrak{R}$ satisfies (5.2a) for all $r_{\tau} \in \mathfrak{R}_{\tau}$. If $s_{1}, s_{2}, \ldots, s_{n}$ and $t_{1}, t_{2}, \ldots, t_{n}$ satisfy the conditions in Lemma 4.3 , then each $t_{i}$ lies in $\mathfrak{R}_{\tau}$. So $c t_{i}=t_{i} c^{\tau}$ by (5.2a). This implies that

$$
\sum_{i=1}^{n} s_{i} c t_{i}=\sum_{i=1}^{n} s_{i} t_{i} c^{\tau}=1 c^{\tau}=c^{\tau} .
$$

Hence (5.2b) holds if (5.2a) does. This proves that there can be at most one $c^{\tau} \in \mathfrak{R}$ satisfying (5.2a). 
There are always some elements $s_{1}, s_{2}, \ldots, s_{n}$ and $t_{1}, t_{2}, \ldots, t_{n}$ satisfying the conditions in Lemma 4.3. We can use these elements to define $c^{\tau} \in \mathfrak{R}$ by $(5.2 \mathrm{~b})$. Since each $s_{i}$ lies in $\mathfrak{R}_{\tau^{-1}}$, its product $r_{\tau} s_{i}$ with any $r_{\tau} \in \mathfrak{R}_{\tau}$ lies in $\mathfrak{R}_{\tau^{\prime}} \mathfrak{R}_{\tau^{-1}}=\mathfrak{R}_{1}$. Hence $r_{\tau} s_{i}$ commutes with $c \in \mathfrak{C}=\mathrm{C}_{\mathfrak{R}}\left(\mathfrak{R}_{1}\right)$. So

$$
r_{\tau} c^{\tau}=\sum_{i=1}^{n} r_{\tau} s_{i} c t_{i}=\sum_{i=1}^{n} c r_{\tau} s_{i} t_{i}=c r_{\tau} 1=c r_{\tau} .
$$

Therefore $c^{\tau}$ satisfies (5.2a), and the lemma is proved.

We call the element $c^{\tau}$ defined in the above lemma the conjugate of $c \in \mathfrak{C}$ by $\tau \in G$. Of course conjugation is an action of $G$ on $\mathfrak{C}$.

Proposition 5.3. If Hypothesis 4.2 holds, then $c^{\tau}$ lies in $\mathfrak{C}$ for any $c \in$ $\mathfrak{C}=\mathrm{C}_{\mathfrak{R}}\left(\Re_{1}\right)$ and any $\tau \in G$. The map $c, \tau \mapsto c^{\tau}$ is then an action of the group $G$ as automorphisms of the ring $\mathfrak{C}$. So it makes $\mathfrak{C}$ a $G$-ring.

Proof. Given $\tau \in G$ we may fix elements $s_{1}, s_{2}, \ldots, s_{n}$ and $t_{1}, t_{2}, \ldots, t_{n}$ satisfying the conditions in Lemma 4.3. If $r_{1} \in \mathfrak{R}_{1}$, then $t_{i} r_{1}$ lies in $\mathfrak{R}_{\tau} \mathfrak{R}_{1}=$ $\mathfrak{R}_{\tau}$ for all $i=1,2, \ldots, n$. This and (5.2a) imply that

$$
c t_{i} r_{1}=t_{i} r_{1} c^{\tau}
$$

for any $c \in \mathfrak{C}$ and $i=1, \ldots, n$. In view of $(5.2 \mathrm{~b})$ it follows that

$$
c^{\tau} r_{1}=\sum_{i=1}^{n} s_{i} c t_{i} r_{1}=\sum_{i=1}^{n} s_{i} t_{i} r_{1} c^{\tau}=1 r_{1} c^{\tau}=r_{1} c^{\tau} \text {. }
$$

So $c^{\tau} \in \mathfrak{R}$ commutes with any $r_{1} \in \mathfrak{R}_{1}$, and hence lies in $\mathfrak{C}=\mathrm{C}_{\mathfrak{R}}\left(\mathfrak{R}_{1}\right)$.

Suppose that $\sigma$ is also an element of $G$. Then (5.2a) says that $r_{\sigma}^{\prime} c^{\sigma}=c r_{\sigma}^{\prime}$ for any $c \in \mathfrak{C}$ and $r_{\sigma}^{\prime} \in \mathfrak{R}_{\sigma}$. Since $c^{\sigma}$ lies in $\mathfrak{C}$ by the above arguments, the element $\left(c^{\sigma}\right)^{\tau}$ is defined and satisfies $r_{\tau}\left(c^{\sigma}\right)^{\tau}=c^{\sigma} r_{\tau}$ for any $r_{\tau} \in \mathfrak{R}_{\tau}$. It follows that

$$
r_{\sigma}^{\prime} r_{\tau}\left(c^{\sigma}\right)^{\tau}=r_{\sigma}^{\prime} c^{\sigma} r_{\tau}=c r_{\sigma}^{\prime} r_{\tau}
$$

for any such $r_{\sigma}^{\prime}$ and $r_{\tau}$. By (4.1) the products $r_{\sigma}^{\prime} r_{\tau}$ generate $\mathfrak{R}_{\sigma \tau}$ as an additive group. So $\left(c^{\sigma}\right)^{\tau}$ satisfies $r_{\sigma \tau}^{\prime \prime}\left(c^{\sigma}\right)^{\tau}=c r_{\sigma \tau}^{\prime \prime}$ for all $r_{\sigma \tau}^{\prime \prime} \in \mathfrak{R}_{\sigma \tau}$. Hence $\left(c^{\sigma}\right)^{\tau}=c^{\sigma \tau}$ by Lemma 5.1. Any element $c \in \mathfrak{C}=\mathrm{C}_{\mathfrak{R}}\left(\mathfrak{R}_{1}\right)$ satisfies $r_{1} c=c r_{1}$ for all $r_{1} \in \mathfrak{R}_{1}$. So $c^{1}=c$ by Lemma 5.1. Therefore the map $c, \tau \mapsto c^{\tau}$ is an action of the group $G$ on the set $\mathfrak{C}$.

If $c, d \in \mathfrak{C}$ and $\tau \in G$, then (5.2a) tells us that

$$
r_{\tau}\left(c^{\tau}+d^{\tau}\right)=r_{\tau} c^{\tau}+r_{\tau} d^{\tau}=c r_{\tau}+d r_{\tau}=(c+d) r_{\tau}
$$


and

$$
r_{\tau}\left(c^{\tau} d^{\tau}\right)=c r_{\tau} d^{\tau}=c d r_{\tau}
$$

for any $r_{\tau} \in \mathfrak{R}_{\tau}$. Applying Lemma 5.1, we conclude that $(c+d)^{\tau}=c^{\tau}+d^{\tau}$ and $(c d)^{\tau}=c^{\tau} d^{\tau}$. Therefore the bijection $c \mapsto c^{\tau}$ is an automorphism of the ring $\mathfrak{C}$, and the proposition is proved.

Conjugation restricts properly to subgroups of $G$.

Proposition 5.4. If Hypothesis 4.2 holds, then the restriction of $\mathfrak{R}$ to any subgroup $H$ of $G$ is a fully $H$-graded ring $\mathfrak{R}[H]$. The centralizer $\mathrm{C}_{\mathfrak{R}[H]}\left(\mathfrak{R}[H]_{1}\right)$ of the identity component in $\mathfrak{R}[H]$ is equal as an $H$-graded ring to the restriction $\mathfrak{C}[H]$ of the centralizer $\mathfrak{C}=\mathrm{C}_{\mathfrak{R}}\left(\mathfrak{R}_{1}\right)$ to $H$. The conjugation action of $H$ on $\mathfrak{C}[H]$, as defined by Lemma 5.1 for the fully $H$-graded ring $\mathfrak{R}[H]$, is the restriction of the conjugation action of $G$ on $\mathfrak{C}$, as defined by Lemma 5.1 for the fully G-graded ring $\mathfrak{R}$.

Proof. It follows from (3.3b) and (4.1) that

$$
\mathfrak{R}[H]_{\sigma} \mathfrak{R}[H]_{\tau}=\mathfrak{R}_{\sigma} \mathfrak{R}_{\tau}=\mathfrak{R}_{\sigma \tau}=\mathfrak{R}[H]_{\sigma \tau}
$$

for any $\sigma, \tau \in H$. Hence $\mathfrak{R}[H]$ is fully $H$-graded.

By (3.6) the $\sigma$-component of $\mathrm{C}_{\mathfrak{R}[H]}\left(\mathfrak{R}[H]_{1}\right)$ is

$$
\mathrm{C}\left(\mathfrak{R}[H]_{1} \text { in } \mathfrak{R}[H]_{\sigma}\right)=\mathrm{C}\left(\mathfrak{R}_{1} \text { in } \mathfrak{R}_{\sigma}\right)=\mathfrak{C}_{\sigma}
$$

for any $\sigma \in H$. It follows that

$$
\mathrm{C}_{\mathfrak{R}[H]}\left(\mathfrak{R}[H]_{1}\right)=\sum_{\sigma \in H} \mathrm{C}\left(\mathfrak{R}[H]_{1} \text { in } \mathfrak{R}[H]_{\sigma}\right)=\sum_{\sigma \in H} \mathfrak{C}_{\sigma}=\mathfrak{C}[H]
$$

as $H$-graded rings.

If $c \in \mathrm{C}_{\mathfrak{R}[H]}\left(\mathfrak{R}[H]_{1}\right)$ and $\tau \in H$, then the $\tau$-conjugate of $c$, as defined by Lemma 5.1 for the fully $H$-graded ring $\mathfrak{R}[H]$, is the unique element $c^{\tau} \in \mathfrak{R}[H]$ such that $r_{\tau} c^{\tau}=c r_{\tau}$ for all $r_{\tau} \in \mathfrak{R}[H]_{\tau}$. Since $\mathfrak{R}[H]_{\tau}$ is equal to $\mathfrak{R}_{\tau}$, this $c^{\tau}$ is also the unique element in $\mathfrak{R}$ such that $r_{\tau} c^{\tau}=c r_{\tau}$ for all $r_{\tau} \in \mathfrak{R}_{\tau}$. So it is the $\tau$-conjugate of $c \in \mathfrak{C}$, as defined by Lemma 5.1 for the fully $G$-graded ring $\mathfrak{R}$. Thus the proposition holds.

The fixed subring under conjugation is easy to compute.

Proposition 5.5. If Hypothesis 4.2 holds, then the fixed subring $\mathfrak{C}^{G}$ of $G$ in $\mathfrak{C}=\mathrm{C}_{\mathfrak{R}}\left(\Re_{1}\right)$ satisfies

$$
\mathfrak{C}^{G}=\mathrm{Z}(\mathfrak{R})=\mathrm{Z}(\mathfrak{C})^{G} \subseteq \mathrm{Z}(\mathfrak{C}) .
$$


Proof. By (5.2a) an element $c \in \mathfrak{C}$ lies in $\mathfrak{C}^{G}$ if and only if it centralizes $\mathfrak{R}_{\tau}$ for every $\tau \in G$. Clearly this happens if and only if $c$ centralizes $\mathfrak{R}=\sum_{\tau \in G} \mathfrak{R}_{\tau}$. Hence $\mathfrak{C}^{G}$ is the intersection $\mathrm{Z}(\mathfrak{R}) \cap \mathfrak{C}$. Since the centralizer $\mathfrak{C}$ of $\mathfrak{R}_{1}$ must contain $\mathrm{Z}(\mathfrak{R})$, this implies that $\mathfrak{C}^{G}=\mathrm{Z}(\mathfrak{R})$. It follows that $\mathfrak{C}^{G}$ is contained in $\mathrm{Z}(\mathfrak{C})$, and so must equal $\mathrm{Z}(\mathfrak{C})^{G}$. Thus the proposition holds.

One elementary consequence of the above proposition and Theorem 2.2 is:

Theorem 5.6. If $G$ is a finite group, then a fully $G$-graded ring $\mathfrak{R}$ has finite block theory if and only if its subring $\mathfrak{C}=\mathrm{C}_{\mathfrak{R}}\left(\mathfrak{R}_{1}\right)$ has finite block theory.

Proof. Suppose that $\mathfrak{R}$ has finite block theory. Then $\mathrm{Z}(\mathfrak{R})$ has finite block theory by Proposition 1.8. Since $\mathfrak{C}^{G}$ is equal to $\mathrm{Z}(\mathfrak{R})$ by Proposition 5.5 , it also has finite block theory. Then $\mathfrak{C}$ has finite block theory by Theorem 2.2.

Conversely, suppose that $\mathfrak{C}$ has finite block theory. Then Proposition 1.4 tells us that $\mathrm{Z}(\mathfrak{C})$ has only a finite number of distinct idempotents. We know from Proposition 5.5 that $Z(\Re)$ is a subring of $Z(\mathfrak{C})$. Hence $Z(\mathfrak{R})$ has only a finite number of distinct idempotents. So $\mathfrak{R}$ has finite block theory by Proposition 1.4, and the theorem is proved.

In fact, we can always describe the blocks of $\mathfrak{R}$ in terms of those of $\mathfrak{C}$ when $G$ is finite, whether or not $\mathfrak{R}$ has finite block theory. The conjugation action of the group $G$ on the ring $\mathfrak{C}$ must permute among themselves the distinct primitive idempotents $1_{C}$, for $C \in \operatorname{Blk}(\mathfrak{C})$, of $\mathrm{Z}(\mathfrak{C})$. So there is an induced conjugation action of $G$ on the set $\operatorname{Blk}(\mathfrak{C})$, with any $\tau \in G$ sending any block $C \in \operatorname{Blk}(\mathfrak{C})$ to the unique conjugate block $C^{\tau}$ of $\mathfrak{C}$ such that

$$
1_{C^{\tau}}=\left(1_{C}\right)^{\tau} .
$$

We write $\mathrm{Blk}_{G}(\mathfrak{C})$ for the set of all $G$-orbits $\mathcal{C}$ in $\operatorname{Blk}(\mathfrak{C})$ under this action.

Theorem 5.8. If $G$ is a finite group, then there is a one to one correspondence between all blocks $B$ of any fully $G$-graded ring $\mathfrak{R}$ and all $G$-orbits $\mathcal{C} \in \mathrm{Blk}_{G}(\mathfrak{C})$ of blocks of the subring $\mathfrak{C}=\mathrm{C}_{\mathfrak{R}}\left(\mathfrak{R}_{1}\right)$ of $\mathfrak{R}$. Two such $B$ and $\mathcal{C}$ correspond if and only if

$$
1_{B}=1_{\mathcal{C}}=\sum_{C \in \mathcal{C}} 1_{C} \in \mathrm{Z}(\mathfrak{C})^{G}=\mathrm{Z}(\mathfrak{R}) .
$$


Proof. Let $B$ be any block of $\mathfrak{R}$. Then $1_{B}$ is a primitive idempotent in $\mathrm{Z}(\mathfrak{R})=\mathfrak{C}^{G}$. It follows that $\mathfrak{C}$ is the direct sum

$$
\mathfrak{C}=\mathfrak{C} 1_{B} \dot{+} \mathfrak{C}\left(1-1_{B}\right)
$$

of $G$-subrings. The fixed subring of the first summand in this decomposition is the indecomposable subring $\left(\mathfrak{C} 1_{B}\right)^{G}=\mathfrak{C}^{G} 1_{B}=\mathrm{Z}(\mathfrak{R}) 1_{B}$. Hence $\left(\mathfrak{C} 1_{B}\right)^{G}$ has finite block theory. Because $G$ is finite, Theorem 2.2 tells us that the $G$-ring $\mathfrak{C} 1_{B}$ has finite block theory.

The decomposition (5.10) implies that the distinct primitive idempotents in $\mathrm{Z}\left(\mathfrak{C} 1_{B}\right)$ are the $1_{C}$, where $C$ runs over the set $\mathcal{C}$ of all blocks $C \in \operatorname{Blk}(\mathfrak{C})$ such that $1_{C}$ lies in $\mathfrak{C} 1_{B}$. Since the $G$-ring $\mathfrak{C} 1_{B}$ has finite block theory, Corollary 1.6 tells us that the distinct idempotents in $\mathrm{Z}\left(\mathfrak{C} 1_{B}\right)$ are just the finite sums

$$
1_{\mathcal{C}^{\prime}}=\sum_{C^{\prime} \in \mathcal{C}^{\prime}} 1_{C^{\prime}}
$$

where $\mathcal{C}^{\prime}$ runs over all subsets of $\mathcal{C}$. It is clear from (5.7) that such a sum $1_{\mathcal{C}^{\prime}}$ is fixed by $G$ if and only if the subset $\mathcal{C}^{\prime}$ is invariant under conjugation by $G$. Because the ring $\left(\mathfrak{C} 1_{B}\right)^{G}=\mathrm{Z}(\mathfrak{R}) 1_{B}$ is indecomposable, its only non-zero idempotent is its identity element $1_{B}$. It follows that $\mathcal{C}$ must have only one non-empty $G$-invariant subset $\mathcal{C}^{\prime}$, and that $1_{\mathcal{C}^{\prime}}$ is equal to $1_{B}$. This forces $\mathcal{C}$ to be a $G$-orbit in $\operatorname{Blk}_{G}(\mathfrak{C})$ such that (5.9) holds.

Conversely, suppose that $\mathcal{C}$ is any $G$-orbit in $\operatorname{Blk}_{G}(\mathfrak{C})$. Then $\mathcal{C}$ is a finite set, and the second equation in (5.9) defines an idempotent $1_{\mathcal{C}}$ in $\mathrm{Z}(\mathfrak{C})^{G}$. The $1_{C}$, for $C \in \mathcal{C}$, are the distinct primitive idempotents in the $G$-subring

$$
\mathrm{Z}(\mathfrak{C}) 1_{\mathcal{C}}=\sum_{C \in \mathcal{C}} \mathrm{Z}(\mathfrak{C}) 1_{C}
$$

of $\mathrm{Z}(\mathfrak{C})$. It follows that the distinct idempotents in $\mathrm{Z}(\mathfrak{C}) 1_{\mathcal{C}}$ are the finite sums $1_{\mathcal{C}^{\prime}}$ in $(5.11)$, where $\mathcal{C}^{\prime}$ runs over all subsets of $\mathcal{C}$. Because $\mathcal{C}$ is a $G$-orbit, the only such $1_{\mathcal{C}^{\prime}}$ which is non-zero and $G$-invariant is $1_{\mathcal{C}}$. Hence the unique non-zero idempotent in $\left(\mathrm{Z}(\mathfrak{C}) 1_{\mathcal{C}}\right)^{G}$ is the identity element $1_{\mathcal{C}}$ of that subring. But Proposition 5.5 implies that

$$
\left(\mathrm{Z}(\mathfrak{C}) 1_{\mathcal{C}}\right)^{G}=\mathrm{Z}(\mathfrak{C})^{G} 1_{\mathcal{C}}=\mathrm{Z}(\mathfrak{R}) 1_{\mathcal{C}}
$$

Therefore $1_{\mathcal{C}}$ is a primitive idempotent in $\mathrm{Z}(\mathfrak{R})$, and there is a unique block $B$ of $\mathfrak{R}$ such that (5.9) holds. 


\section{Orthogonal conjugate idempotents.}

We continue to discuss the centralizer $\mathfrak{C}=\mathrm{C}_{\mathfrak{R}}\left(\mathfrak{R}_{1}\right)$ of the identity component in the fully $G$-graded ring $\mathfrak{R}$ of Hypothesis 4.2. The conjugation action of $G$ must permute among themselves the idempotents of the $G$-ring $\mathfrak{C}$. Let $e$ be one of those idempotents, and $H$ be its stabilizer

$$
H=\left\{\tau \in G \mid e^{\tau}=e\right\}
$$

in $G$. Each left coset $T=H \sigma$ of $H$ in $G$ determines a unique $T$-conjugate $e^{T}$ of $e$ such that

$$
e^{T}=e^{\tau} \in \mathfrak{C}
$$

for all $\tau \in T$. The resulting idempotents $e^{T}$, for $T \in H \backslash G$, are the distinct $G$-conjugates of $e$.

We're going to investigate the case where the $G$-conjugates of $e$ are pairwise orthogonal, i.e., where

$$
e^{S} e^{T}= \begin{cases}e^{S} & \text { if } S=T \\ 0 & \text { if } S \neq T\end{cases}
$$

for any $S, T \in H \backslash G$. So all our present assumptions are collected in:

Hypothesis 6.3. $G$ is a multiplicative group, $\mathfrak{R}$ is a fully $G$-graded ring, $\mathfrak{C}$ is the $G$-ring $\mathrm{C}_{\mathfrak{R}}\left(\mathfrak{R}_{1}\right)$, and $H$ is the stabilizer in $G$ of some idempotent $e \in \mathfrak{C}$ satisfying (6.2).

We begin by discussing the right ideal $e \Re$ of $\mathfrak{R}$.

Lemma 6.4. The right ideal $e \mathfrak{R}$ is projective as both a right $\mathfrak{R}$-module and a right $\mathfrak{R}[H]$-module. It is also an $H \backslash G$-graded $\mathfrak{R}$-module, with the T-component

$$
(e \Re)_{T}=e \Re[T]=e \Re e^{T}
$$

for any $T \in H \backslash G$.

Proof. Since $e \mathfrak{R}$ is a direct summand of the regular right $\mathfrak{R}$-module $\mathfrak{R}=e \mathfrak{R} \dot{+}$ $(1-e) \Re$, it is projective as a right $\mathfrak{R}$-module. We know from Proposition 4.5 that $\mathfrak{R}$ is projective as a right $\mathfrak{R}[H]$-module. Hence so is its direct summand $e \Re$.

If $S \in H \backslash G$ and $\sigma \in S$, then (6.1) and (5.2a) imply that

$$
\mathfrak{R}_{\sigma} e^{S}=\mathfrak{R}_{\sigma} e^{\sigma}=e \mathfrak{R}_{\sigma} .
$$


Hence

$$
\mathfrak{R}[S] e^{S}=\sum_{\sigma \in S} \mathfrak{R}_{\sigma} e^{S}=\sum_{\sigma \in S} e \mathfrak{R}_{\sigma}=e \mathfrak{R}[S] .
$$

This and (6.2) tell us that

$$
e \mathfrak{R}[S] e^{T}=\mathfrak{R}[S] e^{S} e^{T}= \begin{cases}\mathfrak{R}[S] e^{S}=e \mathfrak{R}[S] & \text { if } S=T, \\ 0 & \text { if } S \neq T,\end{cases}
$$

for any $S, T \in H \backslash G$. It follows that

$$
e \mathfrak{R} e^{T}=\sum_{S \in H \backslash G} e \mathfrak{R}[S] e^{T}=e \mathfrak{R}[T]
$$

for any $T \in H \backslash G$, and that

$$
e \Re=\sum_{T \in H \backslash G} e \Re[T]=\sum_{T \in H \backslash G} e \Re e^{T} .
$$

Because the idempotents $e^{T}$, for $T \in H \backslash G$, are pairwise orthogonal, we conclude that $e \mathfrak{R}$ is the direct sum of its additive subgroups $(e \mathfrak{R})_{T}$ defined by (6.5) for any $T \in H \backslash G$. Since

$$
e \mathfrak{R}[T] \Re \Re_{\sigma} \subseteq e_{\mathfrak{R}}[T \sigma]
$$

for any $T \in H \backslash G$ and $\sigma \in G$, this direct sum decomposition is an $H \backslash G$ grading making $e \mathfrak{R}$ an $H \backslash G$-graded $\mathfrak{R}$-module. So the lemma is proved.

Our goal in this section is the surprisingly delicate proof of:

Theorem 6.6. If Hypothesis 6.3 holds, then the idempotent e must lie in $\mathrm{Z}(\Re[H])=\mathfrak{C}[H]^{H}$.

Proof. By Lemma 6.4 the right ideal $e \Re$ is the direct sum

$$
e \Re=\sum_{T \in H \backslash G} e \Re[T]
$$

of its $T$-components $(e \Re)_{T}=e \Re[T]$ for $T \in H \backslash G$. Since right multiplication by any $\sigma \in H$ fixes the coset $H \in H \backslash G$, and permutes among themselves all the other cosets $T \in H \backslash G$, it follows that $e \Re$ is the direct sum

$$
e \Re=e \Re[H] \dot{+} e \mathfrak{R}[G-H]
$$


of its $\mathfrak{R}[H]$-submodules $e \Re[H]$ and

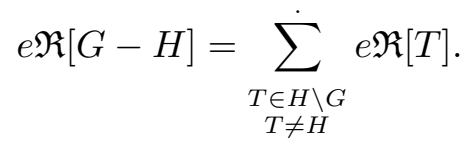

Because $e \Re$ is projective as a right $\mathfrak{R}[H]$-module (see Lemma 6.4), so is its direct summand $e \Re[H]$.

The map $\phi: r \mapsto e r$ is an epimorphism of $\mathfrak{R}[H]$ onto $e \mathfrak{R}[H]$ as right $\mathfrak{R}[H]$ modules. Since $e \mathfrak{R}[H]$ is projective, there is some right ideal $\mathfrak{I}$ in $\mathfrak{R}[H]$ such that

$$
\mathfrak{R}[H]=\mathfrak{I}+\operatorname{ker}(\phi) .
$$

The projection of $\mathfrak{R}[H]$ onto $\mathfrak{I}$ in this decomposition sends $1=1_{\mathfrak{R}}=1_{\mathfrak{R}[H]}$ to an idempotent $f \in \mathfrak{R}[H]$ such that

$$
\mathfrak{I}=f \mathfrak{R}[H] \quad \text { and } \quad \operatorname{ker}(\phi)=(1-f) \mathfrak{R}[H] .
$$

It follows that the epimorphism $\phi$ restricts to an isomorphism $\phi^{\prime}: r \mapsto e r$ of $f \mathfrak{R}[H]$ onto $e \mathfrak{R}[H]$ as right $\mathfrak{R}[H]$-modules. Furthermore, this isomorphism sends $f$ to

$$
e f=\phi^{\prime}(f)=\phi(1)=e 1=e
$$

If $T \in H \backslash G$, then

$$
f \mathfrak{R}[T] \subseteq \mathfrak{R}[H] \mathfrak{R}[T] \subseteq \mathfrak{R}[H T]=\mathfrak{R}[T]
$$

Because $\mathfrak{R}$ is the direct sum of its additive subgroups $\mathfrak{R}[T]$, for $T \in H \backslash G$, it follows that its right ideal $f \Re$ is the direct sum

$$
f \Re=\sum_{T \in H \backslash G} f \mathfrak{R}[T]
$$

of additive subgroups. This $H \backslash G$-grading makes $f \mathfrak{R}$ an $H \backslash G$-graded $\mathfrak{R}$ module, since

$$
f \mathfrak{R}[T] \Re_{\sigma} \subseteq f \mathfrak{R}[T \sigma]
$$

for any $T \in H \backslash G$ and $\sigma \in G$.

In view of $(6.7)$ we have

$$
\text { ef } \mathfrak{R}[T]=e \Re[T]
$$

for each $T \in H \backslash G$. It follows that left multiplication by $e$ is an epimorphism $\Phi^{\prime}: r \mapsto e r$ of $f \mathfrak{R}$ onto $e \mathfrak{R}$ as $H \backslash G$-graded $\mathfrak{R}$-modules. This epimorphism 
restricts to the isomorphism $\phi^{\prime}$ of the $H$-component $f \mathfrak{R}[H]$ of $f \mathfrak{R}$ onto the $H$-component $e \mathfrak{R}[H]$ of $e \Re$. So it must be an isomorphism of $f \mathfrak{R}$ onto $e \mathfrak{R}$ by Proposition 4.7.

Since the $H$-component $e \mathfrak{R}[H]$ of $e \Re$ is equal to $e \Re e^{H}=e \Re e$ by (6.5), the $H$-component $f \mathfrak{R}[H]$ of the isomorphic $H \backslash G$-graded module $f \Re$ must satisfy

$$
f \mathfrak{R}[H]=f \Re e .
$$

In particular, the element $f e=f 1 e$ must lie in $f \mathfrak{R}[H] \subseteq \mathfrak{R}[H]$. But (5.2a) implies that $e$ commutes with every element of $\mathfrak{R}_{\sigma}$ for each $\sigma$ in its stabilizer $H$. Hence $e$ centralizes the direct sum $\mathfrak{R}[H]$ of all those $\mathfrak{R}_{\sigma}$. Because $f$ lies in $\mathfrak{R}[H]$, we conclude from this and (6.7) that $e=e f=f e$ lies in the center of $\mathfrak{R}[H]$. That center is equal to $\mathfrak{C}[H]^{H}$ by Proposition 5.5 , applied to the fully $H$-graded ring $\mathfrak{R}[H]$ in Proposition 5.4. So the theorem is proved.

\section{Morita equivalent subrings.}

Now we're going to assume that the stabilizer $H$ of the idempotent $e$ in Hypothesis 6.3 has finite index in $G$. So $e$ has only a finite number of distinct $G$-conjugates $e^{T}$, for $T \in H \backslash G$. Since these conjugates are pairwise orthogonal by (6.2), their sum

$$
E=\sum_{T \in H \backslash G} e^{T}
$$

is an idempotent in $\mathfrak{C}^{G}$ satisfying

$$
e E=E e=e .
$$

The fixed subring $\mathfrak{C}^{G}$ is equal to $\mathrm{Z}(\mathfrak{R})$ by Proposition 5.5. Hence its idempotent $E$ determines a decomposition of the ring $\mathfrak{R}$ as the direct sum

$$
\mathfrak{R}=\mathfrak{R} E+\mathfrak{R}(1-E)
$$

of subrings. This decomposition allows us to consider $\operatorname{Mod}(\mathfrak{R} E)$ as the full additive subcategory of $\operatorname{Mod}(\mathfrak{R})$ having as objects all right $\mathfrak{R}$-modules $\mathfrak{M}$ such that $\mathfrak{M} E=\mathfrak{M}$.

The idempotent $e$ lies in $\mathrm{Z}(\Re[H])$ by Theorem 6.6. So it determines a decomposition of the ring $\mathfrak{R}[H]$ as the direct sum

$$
\mathfrak{R}[H]=\mathfrak{R}[H] e+\dot{+} \mathfrak{R}[H](1-e)
$$


of subrings. As above, this allows us to consider $\operatorname{Mod}(\mathfrak{R}[H] e)$ to be the full additive subcategory of $\operatorname{Mod}(\mathfrak{R}[H])$ having as objects all right $\mathfrak{R}[H]$-modules $\mathfrak{K}$ satisfying $\mathfrak{K} e=\mathfrak{K}$.

Proposition 7.4. The idempotent e lies in the direct summand $\mathfrak{R} E$ of $\mathfrak{R}$. The direct summand $\mathfrak{R}[H]$ e $=e \mathfrak{R}$ e of $\mathfrak{R}[H]$ is the subring $e(\mathfrak{R} E)$ e of $\mathfrak{R} E$. Furthermore, the two-sided ideal $(\mathfrak{R} E) e(\mathfrak{R} E)=\mathfrak{R} e \mathfrak{R}$ of $\mathfrak{R} E$ is equal to $\mathfrak{R} E$.

Proof. It follows immediately from (7.1b) that $e=e E$ lies in $\mathfrak{R} E$. We know from (6.5) for $T=H$ that $\mathfrak{R}[H] e=e \mathfrak{R}[H]$ is the subring $e \mathfrak{R} e$ of $\mathfrak{R}$. In view of $(7.1 \mathrm{~b})$ this subring is equal to $e(\Re E) e$. Similarly, the two-sided ideal $(\mathfrak{R} E) e(\mathfrak{R} E)$ of $\mathfrak{R} E$ is equal to the two-sided ideal $\mathfrak{R} e \mathfrak{R}$ of $\mathfrak{R}$. If $T \in H \backslash G$ and $\tau \in T$, then

$$
e^{T}=e^{\tau} \in \mathfrak{R}_{\tau^{-1}} e \mathfrak{R}_{\tau} \subseteq \mathfrak{R} e \mathfrak{R}=(\mathfrak{R} E) e(\mathfrak{R} E)
$$

by (6.1) and (5.2b). Hence the ideal $(\mathfrak{R} E) e(\Re E)$ of $\mathfrak{R} E$ contains the sum $E$ in (7.1a). Since $E$ is the identity element of $\mathfrak{R} E$, we conclude that $(\mathfrak{R} E) e(\mathfrak{R} E)=\mathfrak{R} E$. Thus the proposition is proved.

Because $e$ is an idempotent generating $\mathfrak{R} E$ as a two-sided ideal, the two rings $\mathfrak{R}[H] e=e(\mathfrak{R} E) e$ and $\mathfrak{R} E$ are Morita equivalent. We're going to describe the resulting equivalence between the categories $\operatorname{Mod}(\mathfrak{R}[H] e)$ and $\operatorname{Mod}(\mathfrak{R} E)$ in a way that emphasizes its relation with the equivalence between the categories $\operatorname{Mod}(\mathfrak{R}[H])$ and $\operatorname{GrMod}(H \backslash G, \mathfrak{R})$ in Proposition 4.9. All the work is done in two lemmas.

Lemma 7.5. Any right $\mathfrak{R} E$-module $\mathfrak{M}$ is also an $H \backslash G$-graded $\mathfrak{R}$-module, with the T-component $\mathfrak{M} e^{T}$ for any $T \in H \backslash G$. Any homomorphism $\phi: \mathfrak{M} \rightarrow$ $\mathfrak{N}$ of right $\mathfrak{R} E$-modules is also a homomorphism of $H \backslash G$-graded $\mathfrak{R}$-modules. Thus $\operatorname{Mod}(\mathfrak{R} E)$ is naturally a full additive subcategory of $\operatorname{GrMod}(H \backslash G, \mathfrak{R})$. The $H$-component functor $(\cdot)_{H}: \operatorname{GrMod}(H \backslash G, \mathfrak{R}) \rightarrow \operatorname{Mod}(\mathfrak{R}[H])$ sends any object $\mathfrak{M}$ in this subcategory to the $\mathfrak{R}[H]$-submodule $\mathfrak{M}$ e of $\mathfrak{M}$. It also sends any morphism $\phi: \mathfrak{M} \rightarrow \mathfrak{N}$ in this subcategory to its restriction to a morphism $\phi e: \mathfrak{M e} \rightarrow \mathfrak{N e}$ in $\operatorname{Mod}(\mathfrak{R}[H])$. Hence $(\cdot)_{H}$ restricts to an additive functor $(\cdot)$ e sending $\operatorname{Mod}(\Re E)$ into the subcategory $\operatorname{Mod}(\Re[H]$ e) of $\operatorname{Mod}(\Re[H])$.

Proof. The decomposition (7.1a) of $E$ as a finite sum of the pairwise orthogonal idempotents $e^{T}$ gives a decomposition of $\mathfrak{M}=\mathfrak{M} E$ as the finite direct sum

$$
\mathfrak{M}=\sum_{T \in H \backslash G} \mathfrak{M} e^{T}
$$


of additive subgroups. It follows from (6.1) and (5.2a) that

$$
\left(\mathfrak{M} e^{T}\right) \mathfrak{R}_{\sigma}=\mathfrak{M} e^{\tau} \mathfrak{R}_{\sigma}=\mathfrak{M} \mathfrak{R}_{\sigma} e^{\tau \sigma}=\mathfrak{M} e^{T \sigma}
$$

for any $T \in H \backslash G$, any $\tau \in T$, and any $\sigma \in G$. Hence the first statement of the lemma holds.

Clearly any homomorphism $\phi: \mathfrak{M} \rightarrow \mathfrak{N}$ of right $\mathfrak{R} E$-modules sends $\mathfrak{M} e^{T}$ into $\mathfrak{N} e^{T}$ for any $T \in H \backslash G$. So $\phi$ is also a homomorphism of $H \backslash G$-graded $\mathfrak{R}$ modules. Thus the second statement of the lemma holds. This immediately implies the third statement.

The $H$-component functor $(\cdot)_{H}: \operatorname{GrMod}(H \backslash G, \mathfrak{R}) \rightarrow \operatorname{Mod}(\mathfrak{R}[H])$ discussed in $\S 3$ sends any object $\mathfrak{M}$ in the subcategory $\operatorname{Mod}(\mathfrak{R} E)$ to its $H$ component $\mathfrak{M} e^{H}=\mathfrak{M} e$, which certainly lies in $\operatorname{Mod}(\mathfrak{R}[H] e)$. It also sends any morphism $\phi: \mathfrak{M} \rightarrow \mathfrak{N}$ in $\operatorname{Mod}(\mathfrak{R} E)$ to its restriction to a homomorphism $\phi_{H}=\phi e$ of $\mathfrak{M}_{H}=\mathfrak{M} e$ into $\mathfrak{N}_{H}=\mathfrak{N} e$ as right $\mathfrak{R}[H]$-modules. So the remaining statements of the lemma hold.

Lemma 7.6. If $\mathfrak{K}$ is any right $\mathfrak{R}[H]$ e-module, then the $H \backslash G$-graded $\mathfrak{R}$ module $\mathfrak{K} \otimes \mathfrak{R}=\mathfrak{K} \otimes_{\mathfrak{R}[H]} \mathfrak{R}$ is equal to $(\mathfrak{K} \otimes \mathfrak{R}) E$, and hence is also a right $\mathfrak{R} E$-module. The T-component $\mathfrak{K} \otimes \mathfrak{R}[T]$ of $\mathfrak{K} \otimes \mathfrak{R}$ is equal to $(\mathfrak{K} \otimes$ $\mathfrak{R}) e^{T}$ for any $T \in H \backslash G$. So the induction functor $\cdot \otimes \mathfrak{R}: \operatorname{Mod}(\mathfrak{R}[H]) \rightarrow$ $\operatorname{GrMod}(H \backslash G, \mathfrak{R})$ sends the subcategory $\operatorname{Mod}(\mathfrak{R}[H]$ e) of $\operatorname{Mod}(\mathfrak{R}[H])$ into the subcategory $\operatorname{Mod}(\mathfrak{R} E)$ of $\operatorname{GrMod}(H \backslash G, \mathfrak{R})$.

Proof. Of course we regard $\mathfrak{K}$ as a right $\mathfrak{R}[H]$-module using the decomposition (7.3) of $\mathfrak{R}[H]$. Since the idempotent $e$ lies in $\mathfrak{R}[H]$, we have

$$
\mathfrak{K} \otimes \mathfrak{R}[T]=(\mathfrak{K} e) \otimes \mathfrak{R}[T]=\mathfrak{K} \otimes(e \mathfrak{R}[T])
$$

for any $T \in H \backslash G$. It view of (6.5) this last expression is equal to

$$
\mathfrak{K} \otimes\left(e \mathfrak{R} e^{T}\right)=(\mathfrak{K} e) \otimes\left(\mathfrak{R} e^{T}\right)=(\mathfrak{K} \otimes \mathfrak{R}) e^{T},
$$

where all tensor products are over $\mathfrak{R}[H]$. It follows that

$$
\mathfrak{K} \otimes \mathfrak{R}=\sum_{T \in H \backslash G}(\mathfrak{K} \otimes \mathfrak{R}[T])=\sum_{T \in H \backslash G}(\mathfrak{K} \otimes \mathfrak{R}) e^{T}=(\mathfrak{K} \otimes \mathfrak{R}) E .
$$

So the lemma holds.

We can now state the equivalent of the Fong-Reynolds Theorem [F, V.2.5] for our present situation as:

Theorem 7.7. If Hypothesis 6.3 holds with $H$ of finite index in $G$, and if $E$ is the central idempotent $(7.1 \mathrm{a})$ of $\mathfrak{R}$, then the $H$-component functor and the induction functor restrict to additive functors

$(\cdot) e: \operatorname{Mod}(\mathfrak{R} E) \rightarrow \operatorname{Mod}(\mathfrak{R}[H] e) \quad$ and $\cdot \otimes \mathfrak{R}: \operatorname{Mod}(\mathfrak{R}[H] e) \rightarrow \operatorname{Mod}(\mathfrak{R} E)$, 
which form an equivalence between the abelian categories $\operatorname{Mod}(\Re E)$ and $\operatorname{Mod}(\mathfrak{R}[H] e)$.

Proof. This is an immediate consequence of the preceding two lemmas and Proposition 4.9.

Of course the above equivalence between the categories $\operatorname{Mod}(\Re E)$ and $\operatorname{Mod}(\mathfrak{R}[H] e)$ is a Morita equivalence between the rings $\mathfrak{R} E$ and $\mathfrak{R}[H] e$. So it induces an isomorphism between the centers $\mathrm{Z}(\mathfrak{R} E)$ and $\mathrm{Z}(\mathfrak{R}[H] e)$ of those rings. This isomorphism can also be described in a more convenient form. We start with the observation that

Proposition 7.8. The center $\mathrm{Z}(\mathfrak{R} E)=\mathrm{Z}(\mathfrak{R}) E$ is the fixed subring $(\mathfrak{C} E)^{G}=$ $\mathfrak{C}^{G} E$ in the $G$-subring $\mathfrak{C} E$ of $\mathfrak{C}$. The center $\mathrm{Z}(\mathfrak{R}[H] e)=\mathrm{Z}(\mathfrak{R}[H])$ e is the fixed subring $(\mathfrak{C}[H] e)^{H}=\mathfrak{C}[H]^{H}$ e in the $H$-subring $\mathfrak{C}[H]$ e of $\mathfrak{C}[H]$.

Proof. Because the idempotent $E$ lies in $\mathfrak{C}^{G}$, which is contained in $\mathrm{Z}(\mathfrak{C})$ by Proposition 5.5, the $G$-ring $\mathfrak{C}$ is the direct sum

$$
\mathfrak{C}=\mathfrak{C} E \dot{+} \mathfrak{C}(1-E)
$$

of $G$-subrings. Hence $\mathfrak{C}^{G}$ is the direct sum

$$
\mathfrak{C}^{G}=(\mathfrak{C} E)^{G} \dot{+}(\mathfrak{C}(1-E))^{G}
$$

of its subrings $(\mathfrak{C} E)^{G}=\mathfrak{C}^{G} E$ and $(\mathfrak{C}(1-E))^{G}=\mathfrak{C}^{G}(1-E)$. Since $\mathfrak{C}^{G}=\mathrm{Z}(\mathfrak{R})$ by Proposition 5.5, this last decomposition coincides with the decomposition

$$
\mathrm{Z}(\mathfrak{R})=\mathrm{Z}(\mathfrak{R}) E \dot{+} \mathrm{Z}(\mathfrak{R})(1-E)
$$

of $\mathrm{Z}(\mathfrak{R})$ as a direct sum of its subrings $\mathrm{Z}(\mathfrak{R}) E=\mathrm{Z}(\mathfrak{R} E)$ and $\mathrm{Z}(\mathfrak{R})(1-E)=$ $\mathrm{Z}(\mathfrak{R}(1-E))$. So the first statement of the proposition is proved. The second statement is proved similarly, using the fully $H$-graded ring $\mathfrak{R}[H]$, the centralizer $\mathfrak{C}[H]$ of its identity component, and the idempotent $e \in \mathrm{Z}(\mathfrak{R}[H])$ in place of $\mathfrak{R}, \mathfrak{C}$ and $E$, respectively (see Proposition 5.4).

Suppose that $c \in \mathfrak{C}^{H}$ and $T \in H \backslash G$. As in (6.1), we may define the $T$-conjugate $c^{T} \in \mathfrak{C}$ of $c$ to be the common value of the $\tau$-conjugates $c^{\tau}$ for $\tau \in T$. Then the trace map $\operatorname{tr}_{H}^{G}: \mathfrak{C}^{H} \rightarrow \mathfrak{C}^{G}$ is the homomorphism of additive groups sending $c \in \mathfrak{C}^{H}$ to the finite sum

$$
\operatorname{tr}_{H}^{G}(c)=\sum_{T \in H \backslash G} c^{T},
$$


computed in $\mathfrak{C}$. Comparing this with (7.1a), we see that $E$ is the $\operatorname{trace} \operatorname{tr}_{H}^{G}(e)$ of $e \in \mathfrak{C}[H]^{H} \subseteq \mathfrak{C}^{H}$. We use the trace map in the following description of the isomorphism between $\mathrm{Z}(\mathfrak{R} E)$ and $\mathrm{Z}(\mathfrak{R}[H] e)$.

Theorem 7.10. If Hypothesis 6.3 holds with $H$ of finite index in $G$, and if $E$ is the central idempotent $\operatorname{tr}_{H}^{G}(e)$ of $\mathfrak{R}$, then multiplication by $e$ is an isomorphism

$$
\lambda_{e}: z \mapsto z e=e z
$$

of the ring $\mathrm{Z}(\mathfrak{R} E)=\mathfrak{C}^{G} E$ onto the ring $\mathrm{Z}(\Re[H] e)=\mathfrak{C}[H]^{H}$ e. The inverse isomorphism is the restriction of the trace map $\operatorname{tr}_{H}^{G}: \mathfrak{C}^{H} \rightarrow \mathfrak{C}^{G}=\mathrm{Z}(\mathfrak{R})$ to the subring $\mathfrak{C}[H]^{H}$ e of $\mathfrak{C}^{H}$.

Proof. The idempotent $e$ in the subring $\mathrm{Z}(\mathfrak{R}[H])$ of $\mathfrak{R}$ certainly commutes with any element $z \in \mathrm{Z}(\mathfrak{R})$. It follows that multiplication by $e$ is a homomorphism of $\mathrm{Z}(\mathfrak{R})$ into the center of the subring $e \mathfrak{R} e$ of $\mathfrak{R}$. We know from Proposition 7.4 that this subring is equal to $\mathfrak{R}[H]$ e. In view of $(7.1 \mathrm{~b})$ this homomorphism sends $E$ to $E e=e$. Thus its restriction to $\mathrm{Z}(\mathfrak{R} E)=\mathrm{Z}(\mathfrak{R}) E$ is an identity-preserving homomorphism $\lambda_{e}$ of that ring into $\mathrm{Z}(\mathfrak{R}[H] e)$.

By Proposition 7.8 the center $\mathrm{Z}(\mathfrak{R E})$ is the subring $\mathfrak{C}^{G} E$ of $\mathfrak{C}^{G}$. Hence any element $z \in \mathrm{Z}(\mathfrak{R} E)$ lies in $(\mathfrak{C} E)^{H}$ and is fixed under conjugation by any element of either $G$ or $H \backslash G$. Its image $\lambda_{e}(z) \in \mathrm{Z}(\Re[H] e)$ lies in the subring $\mathfrak{C}[H]^{H} e$ of $\mathfrak{C}^{H}$ by Proposition 7.8. So the trace $\operatorname{tr}_{H}^{G}\left(\lambda_{e}(z)\right)$ is defined. It follows from (7.9) and (7.1a) that

$$
\operatorname{tr}_{H}^{G}\left(\lambda_{e}(z)\right)=\sum_{T \in H \backslash G}(z e)^{T}=\sum_{T \in H \backslash G} z^{T} e^{T}=\sum_{T \in H \backslash G} z e^{T}=z E=z .
$$

Thus the restriction of $\operatorname{tr}_{H}^{G}$ is a left inverse to $\lambda_{e}: \mathrm{Z}(\mathfrak{R} E) \rightarrow \mathrm{Z}(\mathfrak{R}[H] e)$.

If $w \in \mathrm{Z}(\mathfrak{R}[H] e)=\mathfrak{C}[H]^{H} e$, then $\operatorname{tr}_{H}^{G}(w)$ lies in $\mathfrak{C}^{G}$ and satisfies

$$
\operatorname{tr}_{H}^{G}(w) E=\operatorname{tr}_{H}^{G}(w) \operatorname{tr}_{H}^{G}(e)=\sum_{S, T \in H \backslash G} w^{S} e^{T} .
$$

Because $w$ is equal to $w e$, it follows from (6.2) that

$$
w^{S} e^{T}=(w e)^{S} e^{T}=w^{S} e^{S} e^{T}= \begin{cases}w^{S} e^{S}=w^{S} & \text { if } S=T, \\ 0 & \text { if } S \neq T,\end{cases}
$$

for any $S, T \in H \backslash G$. Hence

$$
\operatorname{tr}_{H}^{G}(w) E=\sum_{S \in H \backslash G} w^{S}=\operatorname{tr}_{H}^{G}(w) .
$$


So $\operatorname{tr}_{H}^{G}(w)$ lies in $\mathfrak{C}^{G} E=\mathrm{Z}(\mathfrak{R} E)$, and $\lambda_{e}\left(\operatorname{tr}_{H}^{G}(w)\right)$ is defined.

We compute that

$$
\lambda_{e}\left(\operatorname{tr}_{H}^{G}(w)\right)=\operatorname{tr}_{H}^{G}(w) e=\sum_{T \in H \backslash G} w^{T} e .
$$

But $w$ is equal to $w e$, and $e^{T}$ is orthogonal to the idempotent $e=e^{H}$ whenever $T \in H \backslash G$ is different from $H$. Hence

$$
\operatorname{tr}_{H}^{G}(w) e=\sum_{T \in H \backslash G} w^{T} e^{T} e=w^{H} e^{H} e=w e=w .
$$

Therefore $\operatorname{tr}_{H}^{G}: \mathrm{Z}(\mathfrak{R}[H] e) \rightarrow \mathrm{Z}(\mathfrak{R} E)$ is a right inverse to $\lambda_{e}: \mathrm{Z}(\mathfrak{R} E) \rightarrow$ $\mathrm{Z}(\mathfrak{R}[H] e)$, as well as a left inverse. So the theorem is proved.

It remains to be seen that the isomorphism $\lambda_{e}$ of $\mathrm{Z}(\mathfrak{R} E)$ onto $\mathrm{Z}(\mathfrak{R}[H] e)$ in the above theorem is actually the isomorphism $\iota$ associated with the equivalence of $\operatorname{Mod}(\mathfrak{R} E)$ with $\operatorname{Mod}(\mathfrak{R}[H] e)$ in Theorem 7.7. The latter isomorphism can be described as sending any $z \in \mathrm{Z}(\Re E)$ to the unique element $\iota(z) \in \mathrm{Z}(\mathfrak{R}[H] e)$ such that the functor $(\cdot) e$ sends the $\mathfrak{R} E$-endomorphism $m \mapsto m z$ of any right $\mathfrak{R} E$-module $\mathfrak{M}$ to the $\mathfrak{R}[H] e$-endomorphism $k \mapsto k \iota(z)$ of the right $\mathfrak{R}[H] e$-module $\mathfrak{M} e$. Since $(\cdot) e$ is just restriction to $\mathfrak{M} e$, the following observation shows that $\lambda_{e}$ is indeed $\iota$.

Proposition 7.12. If $\mathfrak{M}$ is any right $\mathfrak{R} E$-module, and $z$ is any element of $\mathrm{Z}(\mathfrak{R} E)$, then the $\mathfrak{R}$-endomorphism $m \mapsto m z$ of $\mathfrak{M}$ restricts to the $\mathfrak{R}[H]$ endomorphism $k \mapsto k \lambda_{e}(z)$ of $\mathfrak{M}$.

Proof. Clearly any element $k \in \mathfrak{M} e$ satisfies $k=k e$. It follows from this and (7.11) that

$$
k z=k e z=k \lambda_{e}(z) .
$$

So the proposition holds.

\section{8. $G$-invariant subrings.}

The most natural idempotents $e \in \mathfrak{C}$ satisfying (6.2) are the block idempotents of $G$-invariant subrings of our fully $G$-graded ring $\mathfrak{R}$. By a $G$-invariant subring of $\mathfrak{R}$ we mean a subring $\mathfrak{S}$ of $\mathfrak{R}$ such that

$$
\mathfrak{R}_{\tau^{-1}} \mathfrak{S} \mathfrak{R}_{\tau}=\mathfrak{S}
$$

for every $\tau \in G$. It follows immediately from (4.1) and (3.3a) that:

The restriction of $\mathfrak{R}$ to any normal subgroup $N$ of $G$ is a unitary, G-invariant subring $\mathfrak{R}[N]$ of $\mathfrak{R}$. 
So such subrings always exist.

From now on we fix an arbitrary $G$-invariant subring $\mathfrak{S}$ of $\mathfrak{R}$. For our purposes the most important property of $\mathfrak{S}$ is:

Proposition 8.3. The center $\mathrm{Z}(\mathfrak{S})$ of $\mathfrak{S}$ is a $G$-subring of $\mathfrak{C}=\mathrm{C}_{\mathfrak{R}}\left(\mathfrak{R}_{1}\right)$.

Proof. We first show the the identity element $1_{\mathfrak{S}}$ of $\mathfrak{S}$ lies in $\mathfrak{C}$, i.e., that $\left(1_{\mathfrak{S}}\right) r_{1}=r_{1} 1_{\mathfrak{S}}$ for any $r_{1} \in \mathfrak{R}_{1}$. Since $1_{\mathfrak{R}}$ lies in $\mathfrak{R}_{1}$ by $(3.2)$, it follows from (8.1) for $\tau=1$ that

$$
\left(1_{\mathfrak{S}}\right) r_{1}=\left(1_{\mathfrak{R}} 1_{\mathfrak{S}}\right) r_{1} \in \mathfrak{R}_{1} \mathfrak{S} \mathfrak{R}_{1}=\mathfrak{S}
$$

So

$$
\left(1_{\mathfrak{S}}\right) r_{1}=\left(\left(1_{\mathfrak{S}}\right) r_{1}\right) 1_{\mathfrak{S}}=\left(1_{\mathfrak{S}}\right) r_{1} 1_{\mathfrak{S}}
$$

By left-right symmetry, $r_{1} 1_{\mathfrak{S}}$ is also equal to $\left(1_{\mathfrak{S}}\right) r_{1} 1_{\mathfrak{S}}$, and hence to $\left(1_{\mathfrak{S}}\right) r_{1}$. Thus $1_{\mathfrak{S}}$ lies in $\mathfrak{C}$.

The above argument shows that the product $\left(1_{\mathfrak{S}}\right) r_{1}=r_{1} 1_{\mathfrak{S}}$ lies in $\mathfrak{S}$ for each $r_{1} \in \mathfrak{R}_{1}$. Hence this product commutes with any $z \in \mathrm{Z}(\mathfrak{S})$. It follows that

$$
z r_{1}=\left(z 1_{\mathfrak{S}}\right) r_{1}=z\left(\left(1_{\mathfrak{S}}\right) r_{1}\right)=\left(r_{1} 1_{\mathfrak{S}}\right) z=r_{1}\left(\left(1_{\mathfrak{S}}\right) z\right)=r_{1} z
$$

Therefore $\mathrm{Z}(\mathfrak{S})$ is a subring of $\mathfrak{C}$.

If $z \in \mathrm{Z}(\mathfrak{S})$ and $\tau \in G$, then (5.2b) and (8.1) imply that

$$
z^{\tau}=\sum_{i=1}^{n} s_{i} z t_{i} \in \mathfrak{R}_{\tau^{-1}} \mathfrak{S}_{\mathfrak{R}_{\tau}}=\mathfrak{S}
$$

for some $s_{1}, s_{2}, \ldots, s_{n} \in \mathfrak{R}_{\tau^{-1}}$ and $t_{1}, t_{2}, \ldots, t_{n} \in \mathfrak{R}_{\tau}$. Furthermore, (5.2a) tells us that

$$
z^{\tau} r_{\tau^{-1}}^{\prime} s r_{\tau}=r_{\tau^{-1}}^{\prime}\left(z^{\tau}\right)^{\tau^{-1}} s r_{\tau}=r_{\tau^{-1}}^{\prime} z s r_{\tau}=r_{\tau^{-1}}^{\prime} s z r_{\tau}=r_{\tau^{-1}}^{\prime} s r_{\tau} z^{\tau}
$$

for any $r_{\tau^{-1}}^{\prime} \in \mathfrak{R}_{\tau^{-1}}$, any $s \in \mathfrak{S}$, and any $r_{\tau} \in \mathfrak{R}_{\tau}$. The resulting products $r_{\tau^{-1}}^{\prime} s r_{\tau}$ generate $\mathfrak{S}$ as an additive group by (8.1). Hence $z^{\tau}$ both lies in $\mathfrak{S}$ and centralizes $\mathfrak{S}$. So $z^{\tau}$ lies in $\mathrm{Z}(\mathfrak{S})$, and the proposition is proved.

The conjugation action of $G$ on the $G$-subring $\mathrm{Z}(\mathfrak{S})$ of $\mathfrak{C}$ must permute among themselves the primitive idempotents $1_{C}$, for $C \in \operatorname{Blk}(\mathfrak{S})$, of that subring. As in (5.7), we conclude that there is a natural conjugation action of the group $G$ on the set $\operatorname{Blk}(\mathfrak{S})$, with any $\tau \in G$ sending any block $C$ of $\mathfrak{S}$ to the unique conjugate block $C^{\tau} \in \operatorname{Blk}(\mathfrak{S})$ such that

$$
1_{C^{\tau}}=\left(1_{C}\right)^{\tau} \in \mathrm{Z}(\mathfrak{S}) .
$$


Now we fix a block $C \in \operatorname{Blk}(\mathfrak{S})$ and its stabilizer $G_{C}$ in $G$. So all our current assumptions are gathered in:

Hypothesis 8.5. $G$ is a multiplicative group, $\mathfrak{R}$ is a fully $G$-graded ring, $\mathfrak{S}$ is a $G$-invariant subring of $\mathfrak{R}$, and $C$ is a block of $\mathfrak{S}$ with stabilizer $G_{C}$ in $G$.

The idempotent $1_{C}$ of $\mathrm{Z}(\mathfrak{S})$ lies in $\mathfrak{C}$ by Proposition 8.3. Its distinct $G$ conjugates $\left(1_{C}\right)^{\tau}$ are distinct primitive idempotents $1_{C^{\tau}}$ in $\mathrm{Z}(\mathfrak{S})$, and hence are pairwise orthogonal. So Hypothesis 6.3 holds with $e=1_{C}$ and $H=G_{C}$. This allows us to reproduce the above relations between $G, \mathfrak{R}, \mathfrak{S}$ and $C$ on a smaller scale.

Proposition 8.6. If Hypothesis 8.5 holds, then the restriction $\mathfrak{R}\left[G_{C}\right]$ is a fully $G_{C}$-graded ring. The intersection $\mathfrak{S}_{C}=\mathfrak{R}\left[G_{C}\right] \cap \mathfrak{S}$ is a unitary $G_{C^{-}}$ invariant subring of $\mathfrak{R}\left[G_{C}\right]$. The idempotent $1_{C}$ lies in $\mathrm{Z}\left(\mathfrak{S}_{C}\right)$. Furthermore, the direct summand $\mathfrak{S}_{C} 1_{C}$ of $\mathfrak{S}_{C}$ is equal to the direct summand $\mathfrak{S} 1_{C}$ of $\mathfrak{S}$. Hence there is a unique $G_{C}$-invariant block $C_{C}$ of $\mathfrak{S}_{C}$ such that $1_{C_{C}}=1_{C}$. So Hypothesis 8.5 holds with $G_{C}, \mathfrak{R}\left[G_{C}\right], \mathfrak{S}_{C}$, and $C_{C}$ in place of $G, \mathfrak{R}, \mathfrak{S}$ and $C$, respectively.

Proof. The first statement of the proposition holds by Proposition 5.4. Since $\mathfrak{R}\left[G_{C}\right]$ and $\mathfrak{S}$ are unitary subrings of $\mathfrak{R}$, their intersection $\mathfrak{S}_{C}$ is a unitary subring of each of them. If $\tau \in G_{C}$, then (3.3a), (4.1) and (8.1) imply that

$$
\begin{aligned}
\mathfrak{R}_{\tau^{-1}} \mathfrak{S}_{C} \mathfrak{R}_{\tau}= & \mathfrak{R}_{\tau^{-1}}\left(\mathfrak{R}\left[G_{C}\right] \cap \mathfrak{S}\right) \mathfrak{R}_{\tau} \subseteq \\
& \mathfrak{R}_{\tau^{-1}} \mathfrak{R}\left[G_{C}\right] \mathfrak{R}_{\tau} \cap \mathfrak{R}_{\tau^{-1}} \mathfrak{S}_{\tau}=\mathfrak{R}\left[G_{C}\right] \cap \mathfrak{S}=\mathfrak{S}_{C} .
\end{aligned}
$$

Similarly we have

$$
\mathfrak{R}_{\tau} \mathfrak{S}_{C} \mathfrak{R}_{\tau^{-1}} \subseteq \mathfrak{S}_{C} .
$$

Since $1=1_{\mathfrak{R}}$ lies in $\mathfrak{R}_{1}=\mathfrak{R}_{\tau^{-1}} \mathfrak{R}_{\tau}$ by (3.2) and (4.1), we conclude that

$$
\mathfrak{S}_{C}=1\left(\mathfrak{S}_{C}\right) 1 \subseteq \mathfrak{R}_{\tau^{-1}} \mathfrak{R}_{\tau} \mathfrak{S}_{C} \mathfrak{R}_{\tau^{-1}} \mathfrak{R}_{\tau} \subseteq \mathfrak{R}_{\tau^{-1}} \mathfrak{S}_{C} \mathfrak{R}_{\tau}
$$

Therefore $\mathfrak{R}_{\tau^{-1}} \mathfrak{S}_{C} \mathfrak{R}_{\tau}$ is equal to $\mathfrak{S}_{C}$ for any $\tau \in G_{C}$, and the second statement of the proposition is proved.

Theorem 6.6 for $e=1_{C}$ and $H=G_{C}$ tells us that $1_{C}$ lies in $\mathrm{Z}\left(\mathfrak{R}\left[G_{C}\right]\right)$. Since $1_{C}$ already lies in $\mathrm{Z}(\mathfrak{S})$, this implies that it lies in $\mathrm{Z}\left(\mathfrak{S}_{C}\right)$. The direct summand $\mathfrak{S} 1_{C}=1_{C} \mathfrak{S}$ of $\mathfrak{S}$ is contained in $1_{C} \mathfrak{R} 1_{C}$, which is equal to the direct summand $\mathfrak{R}\left[G_{C}\right] 1_{C}=1_{C} \mathfrak{R}\left[G_{C}\right]$ of $\mathfrak{R}\left[G_{C}\right]$ by (6.5) for $e=1_{C}$ and $T=H=G_{C}$. Hence $\mathfrak{S} 1_{C}$ is contained in $\mathfrak{S}_{C}=\mathfrak{R}\left[G_{C}\right] \cap \mathfrak{S}$. This forces $\mathfrak{S} 1_{C}$ to equal $\mathfrak{S}_{C} 1_{C}$. Thus the third and fourth statements of the proposition hold. 
Because $1_{C}$ is a primitive idempotent in $\mathrm{Z}(\mathfrak{S})$, the ring $\mathfrak{S} 1_{C}$ is indecomposable. Hence so is the equal ring $\mathfrak{S}_{C} 1_{C}$. Therefore $1_{C}$ is a primitive idempotent in $\mathrm{Z}\left(\mathfrak{S}_{C}\right)$. So there is a unique block $C_{C}$ of $\mathfrak{S}_{C}$ such that $1_{C_{C}}=1_{C}$. The $G_{C}$-invariance of $1_{C}$ implies that of both $1_{C_{C}}$ and $C_{C}$. Thus the fifth statement of the proposition holds. The remaining statement follows from the preceding ones.

In order to apply the results in $\S 7$ we must assume that the stabilizer $G_{C}$ has finite index in $G$, i.e., that $C$ belongs to a finite $G$-orbit $\mathcal{C}$ of blocks of $\mathfrak{S}$. Then the equivalent of the idempotent $E$ in (7.1a) is the finite sum

$$
1_{\mathcal{C}}=\sum_{C^{\prime} \in \mathcal{C}} 1_{C^{\prime}}
$$

of pairwise orthogonal idempotents $1_{C^{\prime}}$ in $\mathrm{Z}(\mathfrak{S})$. The decompositions (7.2) and (7.3) now become the decompositions

$$
\mathfrak{R}=\mathfrak{R} 1_{\mathcal{C}}+\mathfrak{R}\left(1-1_{\mathcal{C}}\right)
$$

and

$$
\mathfrak{R}\left[G_{C}\right]=\mathfrak{R}\left[G_{C}\right] 1_{C_{C}}+\mathfrak{R}\left[G_{C}\right]\left(1-1_{C_{C}}\right)
$$

of $\mathfrak{R}$ and $\mathfrak{R}\left[G_{C}\right]$ as direct sums of subrings.

We say that a block $B$ of $\mathfrak{R}$ lies over the block $C$ of $\mathfrak{S}$ if the idempotent $1_{B} 1_{C}=1_{C} 1_{B}$ in $\mathfrak{C}$ is non-zero. In that case we also say that $C$ lies under $B$. We denote by $\operatorname{Blk}(\mathfrak{R} \mid C)$ the set of all blocks $B$ of $\mathfrak{R}$ lying over $C$. Similarly, we say that a block $B^{\prime}$ of $\mathfrak{R}\left[G_{C}\right]$ lies over the block $C_{C}$ of $\mathfrak{S}_{C}$, or that $C_{C}$ lies under $B^{\prime}$, if the idempotent $1_{B^{\prime}} 1_{C_{C}}=1_{C_{C}} 1_{B^{\prime}}$ of $\mathfrak{C}\left[G_{C}\right]$ is non-zero. We denote by $\operatorname{Blk}\left(\mathfrak{R}\left[G_{C}\right] \mid C_{C}\right)$ the set of all blocks $B^{\prime}$ of $\mathfrak{R}\left[G_{C}\right]$ lying over $C_{C}$.

Proposition 8.9. If $C$ belongs to a finite $G$-orbit $\mathcal{C}$ of blocks of $\mathfrak{S}$, then the $1_{B}$, for $B \in \operatorname{Blk}(\mathfrak{R} \mid C)$, are the distinct primitive idempotents in the center $\mathrm{Z}\left(\mathfrak{R} 1_{\mathcal{C}}\right)$ of the summand $\mathfrak{R} 1_{\mathcal{C}}$ in $(8.8 \mathrm{a})$. Furthermore, the $1_{B^{\prime}}$, for $B^{\prime} \in \operatorname{Blk}\left(\Re\left[G_{C}\right] \mid C_{C}\right)$ are the distinct primitive idempotents in the center $\mathrm{Z}\left(\mathfrak{R}\left[G_{C}\right] 1_{C_{C}}\right)$ of the summand $\mathfrak{R}\left[G_{C}\right] 1_{C_{C}}$ in $(8.8 \mathrm{~b})$.

Proof. Fix a block $B \in \operatorname{Blk}(\mathfrak{R})$. The primitive idempotent $1_{B}$ of $\mathrm{Z}(\mathfrak{R})$ must lie in exactly one of the summands $\mathfrak{R} 1_{\mathcal{C}}$ and $\mathfrak{R}\left(1-1_{\mathcal{C}}\right)$ in $(8.8 \mathrm{a})$. If $1_{B}$ lies in $\Re\left(1-1_{\mathcal{C}}\right)$, then $1_{B} 1_{\mathcal{C}}=0$. Since $1_{\mathcal{C}} 1_{C}=1_{C}$ by $(8.7)$, this implies that $1_{B} 1_{C}=1_{B} 1_{\mathcal{C}} 1_{C}=0$. Therefore $B$ does not lie over $C$ when $1_{B}$ lies in $\mathfrak{R}\left(1-1_{C}\right)$. 
On the other hand, if $1_{B}$ lies in $\mathfrak{R} 1_{\mathcal{C}}$, then

$$
0 \neq 1_{B}=1_{B} 1_{\mathcal{C}}=\sum_{C^{\prime} \in \mathcal{C}} 1_{B} 1_{C^{\prime}}
$$

So $B$ lies over some block $C^{\prime} \in \mathcal{C}$. Since $C^{\prime}$ is equal to $C^{\tau}$ for some $\tau \in G$, and $1_{B} \in \mathrm{Z}(\mathfrak{R})=\mathfrak{C}^{G}$ is fixed by $\tau$, we have

$$
\left(1_{B} 1_{C}\right)^{\tau}=\left(1_{B}\right)^{\tau}\left(1_{C}\right)^{\tau}=1_{B} 1_{C^{\prime}} \neq 0
$$

Hence $1_{B} 1_{C} \neq 0$, and $B$ lies over $C$ when $1_{B}$ lies in $\Re 1_{\mathcal{C}}$.

Because the primitive idempotents of $\mathrm{Z}\left(\mathfrak{R} 1_{\mathcal{C}}\right)$ are exactly those primitive idempotents of $Z(\Re)$ which lie in the summand $\mathfrak{R} 1_{\mathcal{C}}$ in $(8.8 \mathrm{a})$, the above arguments prove the first statement in the proposition. The second statement is proved similarly, using $G_{C}, \mathfrak{R}\left[G_{C}\right], \mathfrak{S}_{C}$ and $C_{C}$ in place of $G, \mathfrak{R}, \mathfrak{S}$ and $C$, respectively, as in Proposition 8.6.

Now we can apply Theorem 7.10 to obtain:

Theorem 8.10. If Hypothesis 8.5 holds and $C$ belongs to a finite $G$-orbit $\mathcal{C}$ in $\operatorname{Blk}(\mathfrak{S})$, then multiplication by $1_{C}$ is an isomorphism $\lambda_{C}$ of the ring $\mathrm{Z}\left(\Re 1_{\mathcal{C}}\right)$ onto the ring $\mathrm{Z}\left(\mathfrak{R}\left[G_{C}\right] 1_{C_{C}}\right)$. The inverse isomorphism is a restriction of the trace map from $G_{C}$ to $G$. These isomorphisms induce a bijection of $\operatorname{Blk}(\mathfrak{R} \mid C)$ onto $\operatorname{Blk}\left(\mathfrak{R}\left[G_{C}\right] \mid C_{C}\right)$, sending any block $B$ of $\mathfrak{R}$ lying over $C$ to the unique block $B_{C}$ of $\mathfrak{R}\left[G_{C}\right]$ lying over $C_{C}$ such that

$$
\lambda_{C}\left(1_{B}\right)=1_{B} 1_{C}=1_{B_{C}} \quad \text { and } \quad \operatorname{tr}_{G_{C}}^{G}\left(1_{B_{C}}\right)=1_{B}
$$

Proof. This is an immediate consequence of Proposition 8.9 and of Theorem 7.10 applied to the idempotents $e=1_{C}=1_{C_{C}}$ and $E=1_{\mathcal{C}}$.

We say that a block $B \in \operatorname{Blk}(\Re \mid C)$ is linked by Clifford theory for $C$ to the unique block $B_{C} \in \operatorname{Blk}\left(\Re\left[G_{C}\right] \mid C_{C}\right)$ corresponding to it in the above theorem.

Lemma 8.11. If $B \in \operatorname{Blk}(\Re \mid C)$ is linked to $B_{C} \in \operatorname{Blk}\left(\Re\left[G_{C}\right] \mid C_{C}\right)$ by Clifford theory for $C$, then Hypothesis 6.3 holds with $e=1_{B_{C}}$ and $H=G_{C}$. In this case the idempotent $E$ in (7.1 a) is just $1_{B}$.

Proof. The idempotent $1_{B_{C}}$ is the product $1_{B} 1_{C}$ of two idempotents in $\mathfrak{C}$, and hence lies in $\mathfrak{C}$. Since $1_{B} \in \mathrm{Z}(\mathfrak{R})=\mathfrak{C}^{G}$ is $G$-invariant, we have

$$
\left(1_{B_{C}}\right)^{\tau}=\left(1_{B}\right)^{\tau}\left(1_{C}\right)^{\tau}=1_{B} 1_{C}
$$


for any $\tau \in G$. In particular, $G_{C}$ stabilizes $1_{B_{C}}$. If $\tau \in G-G_{C}$, then $1_{C^{\tau}}$ is orthogonal to $1_{C}$, and commutes with $1_{B} \in \mathrm{Z}(\mathfrak{R})$. Hence

$$
\left(1_{B_{C}}\right)^{\tau} 1_{B_{C}}=1_{B} 1_{C^{\tau}} 1_{B} 1_{C}=1_{B} 1_{C^{\tau}} 1_{C}=0 \neq 1_{B_{C}}
$$

in this case. We conclude that $G_{C}$ is precisely the stabilizer of $1_{B_{C}}$ in $G$, and that the distinct $G$-conjugates of $1_{B_{C}}$ are pairwise orthogonal. Thus the first statement of the lemma holds.

The idempotent corresponding to $E$ in (7.1a) is the trace $\operatorname{tr}_{G_{C}}^{G}\left(1_{B_{C}}\right)$ of the idempotent $1_{B_{C}}$ corresponding to $e$, taken from its stabilizer $H=G_{C}$ to $G$. By Theorem 8.10 this trace sends $1_{B_{C}}$ to $1_{B}$. That is the remaining statement of the lemma.

Now we can state the Fong-Reynolds Theorem 7.7 in a more familiar form.

Theorem 8.12. Suppose that Hypothesis 8.5 holds, and that $C$ belongs to a finite $G$-orbit $\mathcal{C}$ of blocks of $\mathfrak{S}$. If $B \in \operatorname{Blk}(\mathfrak{R} \mid C)$ is linked to $B_{C} \in \operatorname{Blk}\left(\Re\left[G_{C}\right] \mid C_{C}\right)$ by Clifford theory for $C$, then multiplication by $1_{B_{C}}$ and induction from $\mathfrak{R}\left[G_{C}\right]$ to $\mathfrak{R}$ restrict to additive functors

$$
(\cdot) 1_{B_{C}}: \operatorname{Mod}\left(\Re 1_{B}\right) \rightarrow \operatorname{Mod}\left(\Re\left[G_{C}\right] 1_{B_{C}}\right)
$$

and

$$
\cdot \otimes \mathfrak{R}: \operatorname{Mod}\left(\Re\left[G_{C}\right] 1_{B_{C}}\right) \rightarrow \operatorname{Mod}\left(\Re 1_{B}\right),
$$

which form an equivalence between the abelian categories $\operatorname{Mod}\left(\Re 1_{B}\right)$ and $\operatorname{Mod}\left(\mathfrak{R}\left[G_{C}\right] 1_{B_{C}}\right)$.

Proof. In view of Lemma 8.11 this follows immediately from Theorem 7.7 for $e=1_{B_{C}}$ and $E=1_{B}$.

\section{Clifford theory.}

In order to obtain the conclusions of the preceding section for every block $B$ of $\mathfrak{R}$, we shall assume from now on that $G, \mathfrak{R}$ and $\mathfrak{S}$ satisfy:

Hypothesis 9.1. $G$ is a finite multiplicative group, $\mathfrak{R}$ is a fully $G$-graded ring having finite block theory, and $\mathfrak{S}$ is a unitary, $G$-invariant subring of $\mathfrak{R}$.

Then we have:

Proposition 9.2. If Hypothesis 9.1 holds, then any $G$-subring $\mathfrak{D}$ of $\mathfrak{C}=$ $\mathrm{C}_{\mathfrak{R}}\left(\mathfrak{R}_{1}\right)$ has finite block theory. Hence any $G$-invariant subring $\mathfrak{S}^{\prime}$ of $\mathfrak{R}$ has finite block theory. In particular, $\mathfrak{S}$ has finite block theory. 
Proof. Since $\mathfrak{R}$ has finite block theory, Proposition 1.4 tells us that the set $\mathrm{ZI}(\mathfrak{R})$ of all idempotents in $\mathrm{Z}(\mathfrak{R})$ is finite. We know from Proposition 5.5 that $\mathrm{ZI}(\mathfrak{R})$ is the set of all idempotents in $\mathfrak{C}^{G}=\mathrm{Z}(\mathfrak{R})$. If $\mathfrak{D}$ is a $G$-subring of $\mathfrak{C}$, then its fixed subring $\mathfrak{D}^{G}$ is a subring of $\mathfrak{C}^{G}$. Hence $\mathfrak{D}^{G}$ contains only a finite number of distinct idempotents, and so must have finite block theory by Proposition 1.4. Now Theorem 2.2 tells us that $\mathfrak{D}$ has finite block theory.

Let $\mathfrak{S}^{\prime}$ be any $G$-invariant subring of $\mathfrak{R}$. Proposition 8.3 tells us that $\mathrm{Z}\left(\mathfrak{S}^{\prime}\right)$ is a $G$-subring of $\mathfrak{C}$. So $\mathrm{Z}\left(\mathfrak{S}^{\prime}\right)$ has finite block theory by the above arguments. Then $\mathfrak{S}^{\prime}$ has finite block theory by Proposition 1.8. Thus the proposition holds.

We denote by $\mathrm{Blk}_{G}(\mathfrak{S})$ the set of all $G$-orbits under the conjugation action (8.4) of $G$ on $\operatorname{Blk}(\mathfrak{S})$. We write $1_{\mathcal{C}}$ for the idempotent associated with any orbit $\mathcal{C} \in \mathrm{Blk}_{G}(\mathfrak{S})$ by $(8.7)$.

Proposition 9.3. If Hypothesis 9.1 holds, the the ring $\mathfrak{R}$ is the direct sum

$$
\mathfrak{R}=\sum_{\mathcal{C} \in \mathrm{Blk}_{G}(\mathfrak{S})} \mathfrak{R} 1_{\mathcal{C}}
$$

of $G$-invariant subrings $\mathfrak{R} 1_{\mathcal{C}}$, each of which has finite block theory.

Proof. We know from Proposition 9.2 that $\mathfrak{S}$ has finite block theory. Since $\mathfrak{S}$ is also a unitary subring of $\mathfrak{R}$ (see Hypothesis 9.1), it follows from (1.3) that its identity element $1_{\mathfrak{R}}$ is the finite sum

$$
1_{\Re}=\sum_{C \in \operatorname{Blk}(\mathfrak{S})} 1_{C}
$$

of pairwise orthogonal primitive idempotents in $\mathrm{Z}(\mathfrak{S})$. The finite set $\mathrm{Blk}(\mathfrak{S})$ is the disjoint union of its $G$-orbits $\mathcal{C} \in \operatorname{Blk}_{G}(\mathfrak{S})$. So $1_{\mathfrak{R}}$ is also the finite sum

$$
1_{\mathfrak{R}}=\sum_{\mathcal{C} \in \operatorname{Blk}_{G}(\mathfrak{S})} 1_{\mathcal{C}}
$$

of pairwise orthogonal idempotents

$$
1_{\mathcal{C}}=\sum_{C \in \mathcal{C}} 1_{C}
$$

Each of these last idempotents lies in $\mathfrak{C}^{G}$, which is equal to $\mathrm{Z}(\mathfrak{R})$ by Proposition 5.5. So this last decomposition of $1_{\mathfrak{R}}$ implies that $\mathfrak{R}$ is the direct sum (9.4) of subrings. 
The idempotent $1_{\mathcal{C}} \in \mathrm{Z}(\mathfrak{R})$ commutes with $\mathfrak{R}_{\tau}$ for any $\mathcal{C} \in \mathrm{Blk}_{G}(\mathfrak{S})$ and $\tau \in G$. Hence

$$
\mathfrak{R}_{\tau^{-1}}\left(\mathfrak{R} 1_{\mathcal{C}}\right) \mathfrak{R}_{\tau}=\left(\mathfrak{R}_{\tau^{-1}} \mathfrak{R} \mathfrak{R}_{\tau}\right) 1_{\mathcal{C}}=\mathfrak{R} 1_{\mathcal{C}}
$$

So $\mathfrak{R} 1_{\mathcal{C}}$ is a $G$-invariant subring of $\mathfrak{R}$. As such it has finite block theory by Proposition 9.2. Thus the proposition holds.

Recall from the preceding section that a block $C$ of $\mathfrak{S}$ lies under a block $B$ of $\mathfrak{R}$ if the idempotent $1_{B} 1_{C}=1_{C} 1_{B}$ in $\mathfrak{R}$ is not zero. The final step in Clifford theory for blocks is:

Theorem 9.5. If Hypothesis 9.1 holds, then the blocks of $\mathfrak{S}$ lying under a fixed block $B \in \operatorname{Blk}(\Re)$ form a single $G$-orbit $\mathcal{C} \in \operatorname{Blk}_{G}(\mathfrak{S})$. This orbit $\mathcal{C}$ is determined by the fact that $1_{B}$ lies in the direct summand $\mathfrak{R} 1_{\mathcal{C}}$ in (9.4).

Proof. The primitive central idempotent $1_{B}$ of $\mathfrak{R}$ must lie in exactly one of the direct summands $\mathfrak{R} 1_{\mathcal{C}}$ in (9.4). Thus there is a unique $G$-orbit $\mathcal{C} \in \operatorname{Blk}_{G}(\mathfrak{S})$ such that $1_{B} \in \mathfrak{R} 1_{\mathcal{C}}$. Then Proposition 8.9 tells us that $B$ lies over every block $C \in \mathcal{C}$ and over no other block of $\mathfrak{S}$. So the theorem holds.

Of course Theorems 8.10 and 8.12 now hold for any block $B$ of $\Re$ and any block $C$ of $\mathfrak{S}$ lying under $B$. In particular, $B$ is linked by Clifford theory for $C$ to a unique block $B_{C}$ of the restriction $\mathfrak{R}\left[G_{C}\right]$ of $\mathfrak{R}$ to the stabilizer $G_{C}$ of $C$. Furthermore, the abelian categories $\operatorname{Mod}\left(\Re 1_{B}\right)$ and $\operatorname{Mod}\left(\Re\left[G_{C}\right] 1_{B_{C}}\right)$ are Morita equivalent.

\section{Questions.}

The above discussion raises a couple of interesting questions which we cannot answer. There is a natural duality between $G$-rings and $G$-graded rings, based on the fact that both are special cases of $\mathfrak{H}$-rings in the sense of [CS], where the Hopf algebra $\mathfrak{H}$ is the group ring $\mathbb{Z} G$ in the former case and its dual in the latter one. This allows us to formulate the dual statement to Theorem 2.2 in the form of:

Question 10.1. If $G$ is a finite group and $\mathfrak{R}$ is any $G$-graded ring whose identity component $\mathfrak{R}_{1}$ has finite block theory, does $\mathfrak{R}$ have finite block theory?

Surprisingly enough, the answer to this question is yes when $G$ has order 2 or 3. This can be shown by direct and brutal calculation of all possible central idempotents in $\mathfrak{R}$. However the computations required for this approach become impossibly complicated as the order of $G$ increases. So any attempt 
to give a positive answer to this question for all $G$ must be based on some other idea.

Rings graded by a finite abelian group $G$ behave very much like $G^{*}$-rings, where $G^{*}$ is the finite dual group to $G$. So the fact that Question 10.1 has a positive answer for a few small abelian groups may be an accident. Before raising this question to the status of a conjecture, it would be wise to see a few non-commutative examples.

One possible consequence of a positive answer to Question 10.1 is:

Proposition 10.2. Suppose that $G$ is a finite group and that $\mathfrak{R}$ is a fully $G$-graded ring with finite block theory. If the answer to Question 10.1 is always yes, then the restriction $\mathfrak{R}[H]$ of $\mathfrak{R}$ to any subgroup $H$ of $G$ also has finite block theory.

Proof. The identity component $\mathfrak{R}_{1}$ is a $G$-invariant subring of $\mathfrak{R}$ by (8.2), and so has finite block theory by Proposition 9.2. A positive answer to Question 10.1 for the $H$-graded ring $\mathfrak{R}[H]$ would then imply the proposition.

Of course, the conclusion of the above proposition might hold even if the answer to Question 10.1 turns out to be no in general.

It is somewhat annoying that we need to assume that the fully $G$-graded ring $\mathfrak{R}$ has finite block theory in order to show that each of its blocks $B$ lies over a single $G$-orbit of blocks of a $G$-invariant subring $\mathfrak{S}$ (see Theorem 9.5). In view of the relations between blocks of $\mathfrak{R}$ and $G$-orbits of blocks of $\mathfrak{C}$ in Theorem 5.8, we might hope that this global assumption can be avoided, i.e., that we have a positive answer to:

Question 10.3. If $G$ is a finite group and $\mathfrak{S}$ is a $G$-invariant subring of a fully $G$-graded ring $\mathfrak{R}$, does every block $B$ of $\mathfrak{R}$ lie over a $G$-conjugacy class $\mathcal{C}$ of blocks of $\mathfrak{S}$ ?

We don't have a single example illustrating this question in a situation where $\mathfrak{R}$ does not have finite block theory. So it is much more speculative than the preceding question. We should remark that the problem is to find one block $C$ of $\mathfrak{S}$ lying under a given block $B$ of $\mathfrak{R}$. Once such a $C$ exists, the rest is easy.

\section{References}

[B] G. Birkhoff, Lattice Theory (third edition), AMS Coll. Publ., 25, Am. Math. Soc. , Providence, 1967. 
[CS] S. Chase and M. Sweedler, Hopf algebras and Galois theory, Springer Lecture Notes in Math., 97, Springer Verlag, Berlin-Heidelberg-New York, 1969.

[D] E. Dade, The equivalence of various generalizations of group rings and modules, Math. Z., 181 (1982), 335-344.

[DRTW] E. Dade, D. Robinson, O. Taussky and M. Ward, Divisors of recurrent sequences, J. reine Angew. Math., 214/215 (1964), 180-183.

[DT1] E. Dade and O. Taussky, On the different in orders in an algebraic number field and special units connected with it, Acta Arith., 9 (1964), 47-51.

[DT2] - Some new results connected with matrices of rational integers, AMS Proc. Symp. Pure Math., 8 (1965), 78-88.

[DTZ] E. Dade, O. Taussky and H. Zassenhaus, On the theory of orders, in particular on the semi-group of ideal classes and genera of an order in an algebraic number field, Math. Annalen, 148 (1962), 31-64.

[E] H. Ellers, Cliques of irreducible representations of $p$-solvable groups and a theorem analogous to Alperin's conjecture, Math. Z., 217 (1994), 607-634.

[F] W. Feit, The representation theory of finite groups, North-Holland, AmsterdamNew York-Oxford, 1982.

[NRvO] C. Năstăsescu, S. Raianu and F. van Oystaeyen, Modules graded by G-sets, Math. Z., 203 (1990), 605-627.

[U] K.-H. Ulbrich, Vollgraduierte Algebren, Abh. Math. Sem. Univ. Hamburg, 51 (1981), 136-148.

This research was supported by grant number DMS 96-00106 from the National Science Foundation.

UNIVERSITY OF ILLINOIS AT URBANA-CHAMPAIGN

1409 W. Green ST.

URBANA, IL 61801, USA

E-mail address: e-dade@uiuc.edu 